\title{
Effekt: Capability-passing style for type- and effect-safe, extensible effect handlers in Scala
}

\author{
JONATHAN IMMANUEL BRACHTHÄUSER(D, PHILIPP SCHUSTER \\ and KLAUS OSTERMANN \\ University of Tübingen, Tübingen, Germany \\ (e-mails: jonathan.brachthaeuser@uni-tuebingen.de, philipp.schuster@uni-tuebingen.de, \\ klaus.ostermann@uni-tuebingen.de)
}

\begin{abstract}
Effect handlers are a promising way to structure effectful programs in a modular way. We present the Scala library Effekt, which is centered around capability passing and implemented in terms of a monad for multi-prompt delimited continuations. Effekt is the first library implementation of effect handlers that supports effect safety and effect polymorphism without resorting to type-level programming. We describe a novel way of achieving effect safety using intersection types and path-dependent types. The effect system of our library design fits well into the programming paradigm of capability passing and is inspired by the effect system of Zhang \& Myers (2019, Proc. ACM Program. Lang. 3(POPL), 5:1-5:29). Capabilities carry an abstract type member, which represents an individual effect type and reflects the use of the capability on the type level. We represent effect rows as the contravariant intersection of effect types. Handlers introduce capabilities and remove components of the intersection type. Reusing the existing type system of Scala, we get effect subtyping and effect polymorphism for free.
\end{abstract}

\section{Introduction}

To get a first impression of Effekt, consider the following piece of code written in Scala and using our library. It models a coin toss, but with a twist: the gambler might be too drunk and lose the coin (Kammar et al., 2013). The program uses two effect operations: flip and raise. The effect operation $f l i p$ is used to nondeterministically decide whether the gambler is too drunk to catch the coin. In that case, we use the effect operation raise to signal an exception. Otherwise, we return the result of a second coin toss as a string.

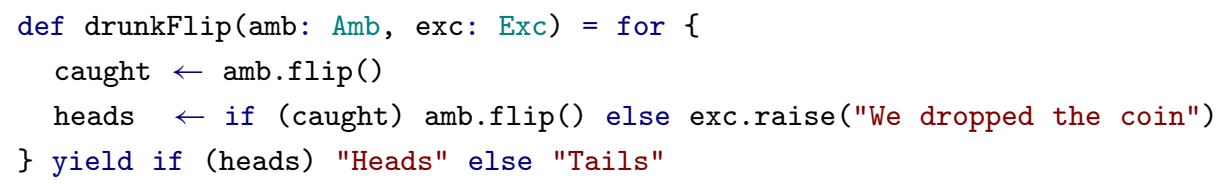


This simple example already shows a few things. Firstly, the program is written in monadic style using Scala's for-comprehensions. ${ }^{1}$ Even though the program uses multiple effects, all effectful code only uses one monad - a variant of the continuation monad. Secondly, the effect operations are methods on capabilities amb and exc, which drunkFlip receives as arguments. The semantics of the effect operations is thus dependent on the corresponding implementations of Amb and Exc. We can, for instance, run the method drunkFlip with the handlers maybe and collect:

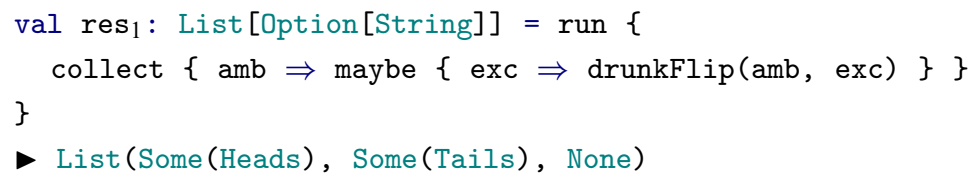

The collect handler enumerates all possible outcomes of the flip operation and collects them in a list. The maybe handler returns None if the program raises an exception.

Swapping the two handlers changes the result type and the semantics:

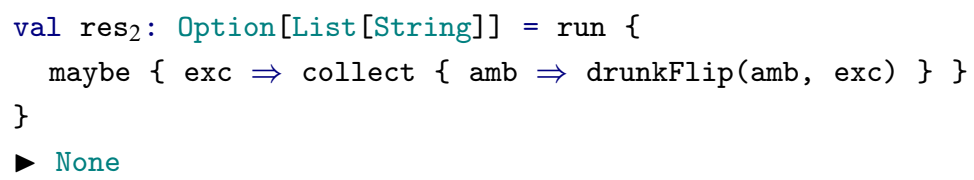

This illustrates an important feature of effect handlers. Programs that use effects are agnostic of the concrete handlers and their order (Plotkin \& Pretnar, 2009). This gives the caller of the program and the implementer of the handlers more flexibility. Moreover, effect handlers are powerful enough to express many different control-flow abstractions as libraries, which otherwise have to be built into a language. Examples are async-await, cooperative multitasking, iterators, exceptions, and many more (Wu et al., 2014; Leijen, 2016, 2017b; Dolan et al., 2017). We encounter some of these abstractions in the remainder of this paper.

\subsection{Effekt - Effect handlers and capability passing}

In this paper, we present Effekt: a library for programming with effect handlers in the language Scala. The combination of effect handlers with object-oriented features enables new modularization strategies, both for effectful programs and for effect handler implementations. While Scala conveniently provides us with all necessary features, the core ideas behind Effekt (passing effect handlers explicitly as capabilities, combining effect handlers with object-oriented programming, and using path-dependent types and intersection types for effect safety) are independent of our embedding into Scala. The different aspects of our library design are summarized in the type signature of the method drunkFlip that we have seen above:

1 Similar to Haskell's do-notation, for-comprehensions in Scala syntactically simplify writing monadic code. In general, for $\left\{\mathrm{x}_{1} \leftarrow \mathrm{e}_{1} ; \mathrm{x}_{2} \leftarrow \mathrm{e}_{2} ; \ldots \mathrm{xn} \leftarrow\right.$ en $\}$ yield $\mathrm{e}$ desugars to $e_{1}$.flatMap $\left\{x_{1} \Rightarrow e_{2}\right.$.flatMap $\left\{x_{2} \Rightarrow \ldots\right.$ en.map $\left.\{x n \Rightarrow e\}\right\}$. 


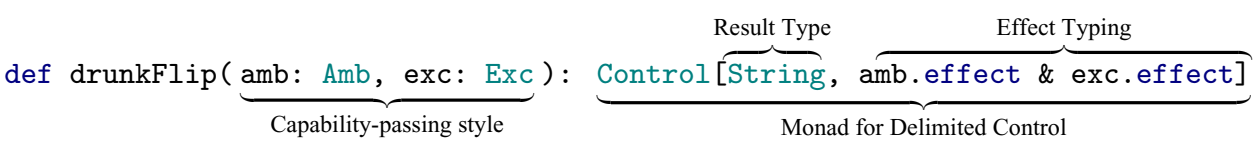

Like previous versions of Effekt (Brachthäuser \& Schuster, 2017), our library design centers around the concept of capability passing. As we will see in the remainder of this paper, capabilities in Effekt encapsulate three different things:

Capabilities contain Effect Implementations. They give semantics to effect operations (Section 2). In the example program drunkFlip, we call effect operations as methods on the capabilities amb and exc, for instance.

Capabilities contain Scope Delimiters. Effect operations can capture the continuation delimited by the corresponding handler. Programs written with our library have type Control (Section 3), a monadic implementation of delimited control with first-class prompts (Dybvig et al., 2007). Prompts act as markers and are used to delimit the scope of continuation capture. Capabilities can close over scope delimiters.

Capabilities contain Effect Labels. Our capabilities contain a type member (amb.effect) that we use as a label on the type level to guarantee effect safety (Section 4). We keep track of all used capabilities by aggregating their effect members in an intersection type as the second-type parameter of Control.

\subsection{Effect system}

Previous versions of Effekt lacked effect safety (Brachthäuser \& Schuster, 2017). Capabilities could be used outside of the scope of their handler region, which resulted in runtime exceptions. In this paper, we solve the problem of effect safety and present a variant of Effekt that statically prevents programs from using leaked capabilities. We describe a novel way of achieving effect safety in a library embedding using intersection types and path-dependent types. The effect system of our library design fits well into the programming paradigm of capability passing and is inspired by the effect system of the $\lambda_{\text {s }}$-calculus by Zhang \& Myers (2019). As we will see in Section 4, we represent effect rows as the contravariant intersection of effect types, where an individual effect of a capability $\mathrm{c}$ is represented by the abstract type member c. effect. Handlers remove components of the intersection type. By reusing the existing type system of Scala, we obtain the features of effect subtyping and effect polymorphism for free. By embedding Effekt into a practical, but unsound host language (Amin \& Tate, 2016), type and effect safety of our library can only be guaranteed up to soundness of the host language. We do not present formal proofs, but a design for a library implementation as an embedding into an existing, mainstream programming language. Nevertheless, from our experience of working with Effekt, we are confident that it inherits the following properties from $\lambda_{\Omega \text { 仓 }}$. 
Effect Safety. Our effect system asserts that all effects are handled. For example, we can only call run on a program when all effects are handled and we reject programs like run $\{$ amb.flip() \}. Like with systems based on monadic regions (Launchbury \& Sabry, 1997; Moggi \& Sabry, 2001; Kiselyov \& Shan, 2008), capabilities can leave their defining handler scope but our effect system ensures that they cannot be used outside their defining handler scope.

Effect Subtyping. We use Scala's support for subtyping of intersection types to implement effect subtyping. A program with type Control[Int, exc.effect] can be used where a program of type Control [Int, exc.effect \& amb.effect] is expected.

Effect Polymorphism. We use Scala's support for type polymorphism to express effect polymorphic functions like:

$$
\operatorname{def} \operatorname{mapM}[A, B, E](1 s: \operatorname{List}[A], f: A \Rightarrow \operatorname{Control}[B, E]) \text { : Control[List [B], E] }
$$

The function maps an effectful functions over a list. It is polymorphic in the effects $\mathrm{E}$ used by function $f$. The effects of mapM are precisely the effects of $f$. Also, mapM may itself use effects and handle them. They are encapsulated and do not appear in the signature.

Existing implementations of languages with effect handlers either completely lack a static effect system - this includes Multicore OCaml (Dolan et al., 2014), Eff (Bauer \& Pretnar, 2015), embeddings of Eff in OCaml (Kiselyov \& Sivaramakrishnan, 2016), and previous versions of Effekt (Brachthäuser \& Schuster, 2017; Brachthäuser et al., 2018) —or they do not have sufficient support for effect polymorphism (Kammar et al., 2013; Inostroza \& van der Storm, 2018). Languages and libraries with effect systems like Extensible Effects (Kiselyov et al., 2013), Koka (Leijen, 2017c), Links (Hillerström et al., 2017), Frank (Lindley et al., 2017), and Helium (Biernacki et al., 2019) require explicit lifting annotations to encapsulate effects in effectful higher-order functions, like the function mapM above. Without such manual liftings, the implementation detail of effects used within mapM would leak into its type signature. In contrast, Effekt requires no such manual lifting.

Related to effect encapsulation is the property of effect parametricity (Zhang \& Myers, 2019). Just by looking at the type of mapM above, we can guarantee that no implementation of mapM can (accidentally or purposefully) handle effects $E$ used by $f$. Handling an effect means two things: delimiting captured continuations and providing an implementation for effect operations. By employing capability passing, Effekt guarantees that no matter how mapM is implemented - it will never change the implementations of effect operations used in $f$. However, it is possible for mapM to instantiate delimit continuations for effects $E$, which are captured within $f$. This breaks effect parametricity as we explain in Section 5.4 in more detail.

\subsection{List of contributions and paper overview}

In summary, this article makes the following contributions:

- We introduce the library design of Effekt, which is based on implementing effects in terms of other effects. In particular, continuations are captured using the Scope 
effect, which serves as a base case. The corresponding built-in effect handler for Scope delimits the extent of the captured continuation.

- We implement Effekt building on the operational semantics of Dybvig et al. (2007). We achieve effect safety by generalizing techniques of Launchbury \& Sabry (1997) to nested regions (Kiselyov \& Shan, 2008) and using intersection types of abstract type members (Parreaux et al., 2017) instead of rank-2 types. Our effect system rules out some use cases of multi-prompt delimited control (Kobori et al., 2016), but can express many interesting use cases of effect handlers.

- We present our API design of ambient state (Kiselyov et al., 2006; Leijen, 2018) as the second of the two built-in effects: the state effect.

- We evaluate the usability of our library in multiple extended case studies.

- We evaluate desirable properties like effect safety and effect parametricity of our library embedding and discuss limitations.

- We evaluate the extensibility properties of our library design. We discuss interesting opportunities to explore type- and effect-safe modularization of effectful programs, opened up by embedding Effekt into Scala, a language that combines functional programming with object-oriented programming.

The remainder of the paper is structured as follows. In Section 2, we give an overview of programming with Effekt and show how to implement effect handlers. While ultimately we aim to achieve an effect-safe implementation of effect handlers, as an intermediate step, we present a monadic implementation of delimited control (Section 3) to then show how to restrict it to an effect-safe, yet useful subset (Section 4). We discuss properties and limitations of our effect system in Section 5. In Section 6, we give several extended case studies and discuss novel extensibility properties that arise from our embedding into Scala. Section 7 discusses related work and Section 8 concludes.

\section{Programming with effect handlers in Effekt}

To introduce programming with effect handlers in our library Effekt, we continue to use the effects from the introduction as a running example. We will see how to declare and handle the exception and ambiguity effects. This section should give the reader a high-level intuition for the usage of the library. The examples of this section have been presented in similar form in Koka (Leijen, 2017c) and previous versions of Effekt for Scala (2017) and Java (2018). All code is given in Dotty (version 0.19), the upcoming version of the Scala programming language. The library and examples from this paper are available online:

\section{https://github.com/b-studios/scala-effekt/tree/jfp}

Programming with effect handlers encourages modularity by separating the interface of an effect (the effect signature) from its implementation (the effect handler). This is reminiscent of how programs are organized in object-oriented programming. In fact, embedding effect handlers into Scala - a language with support for functional as well as objectoriented paradigms - we actively use the correspondence between effect handlers and object-oriented programming (summarized in Figure 1). 


\begin{tabular}{ll} 
Effect Handlers & Object-Oriented Programming \\
\hline Effect Signatures & Interfaces \\
Effect Operation & Method \\
Effect Handlers & Implementations / Classes \\
Effectful Programs & Interface Users \\
Effect Capability & Instances / Objects
\end{tabular}

Fig. 1. Mapping concepts from effect handlers to object-oriented programming.

\subsection{Example: Exceptions—aborting the computation}

Exceptions are a simple effect and provide a good opportunity to introduce the involved concepts. Our effect system guarantees that all effects are handled, which means that the exceptions we implement are checked. This is not the case for Scala's native exceptions they are unchecked.

Scala Background. In Scala, traits not only have value members, but also type members. Value members and type members can be left abstract and defined in implementing classes (Odersky \& Zenger, 2005b). The syntax $x \Rightarrow$ EXPR introduces a lambda that binds $\mathrm{x}$ in EXPR. Methods with one argument (like map) can be written infix (i.e., c map $f$ desugars to $c \cdot \operatorname{map}(f))$. Methods with only one argument can be called with braces, instead of parenthesis, that is, $c \cdot \operatorname{map}(f)$ and $c \cdot \operatorname{map}\{f\}$ are equivalent for the purpose of this paper.

\subsubsection{Effect signatures are interfaces}

Effect signatures group multiple effect operations under one type. In Effekt, we represent effect signatures as interfaces (called "traits" in Scala). Figure 2(a) declares the effect signature Exc. The return type of effect operation raise makes use of the type alias:

type $/[+$ Result, - Effects] = Control[Result, Effects]

In Scala, type constructors with two arguments can be used infix and we write $R / E$ to denote the type Control $[R, E]$. We read $R / E$ as the type of a computation with result type $R$, using effects $E$. The variance annotations + and - make it explicit that effectful computations are covariant in their result type and contravariant in their effects. The return type of effect operations like raise not only tells us the type of the result, in this case Nothing (the bottom of Scala's subtyping lattice). It also describes which effects an effect operation may use, in this case the abstract type member effect (which is short for this.effect). The type member effect is declared in the library trait Eff, that Exc inherits from:

trait Eff $\{$ type effect $\}$

We only use effect types like effect for effect safety. They do not have any operational meaning attached to them and are erased by the compiler. 


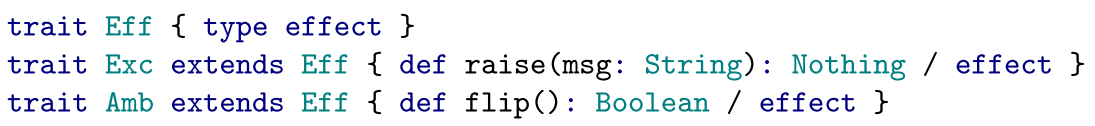

(a) Effect signatures for exception and ambiguity as traits extending library trait Eff.

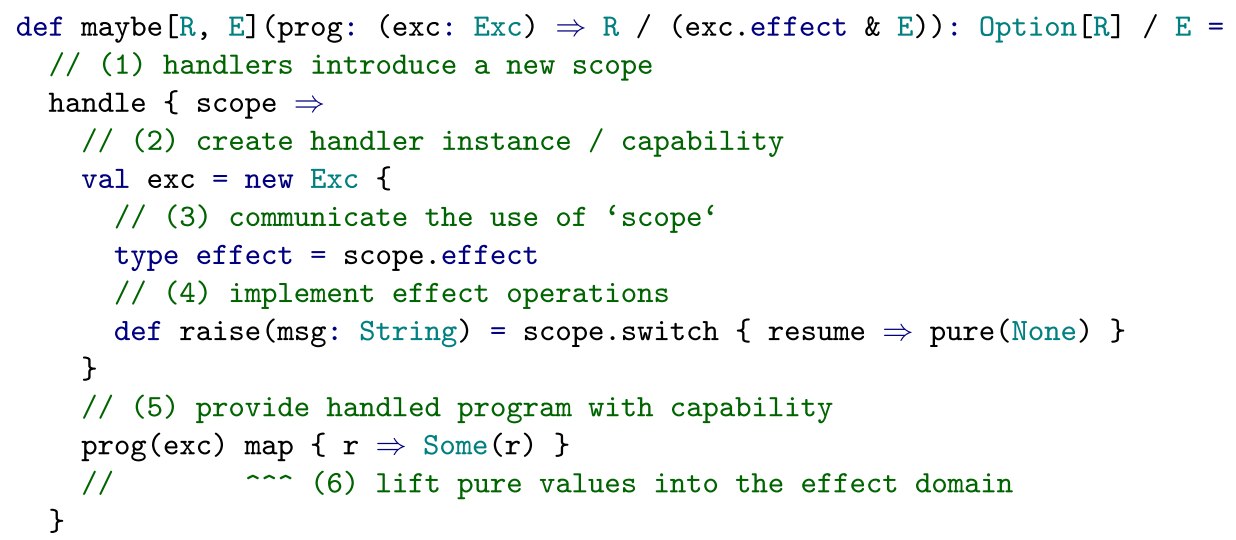

(b) Handler function for the exception effect.

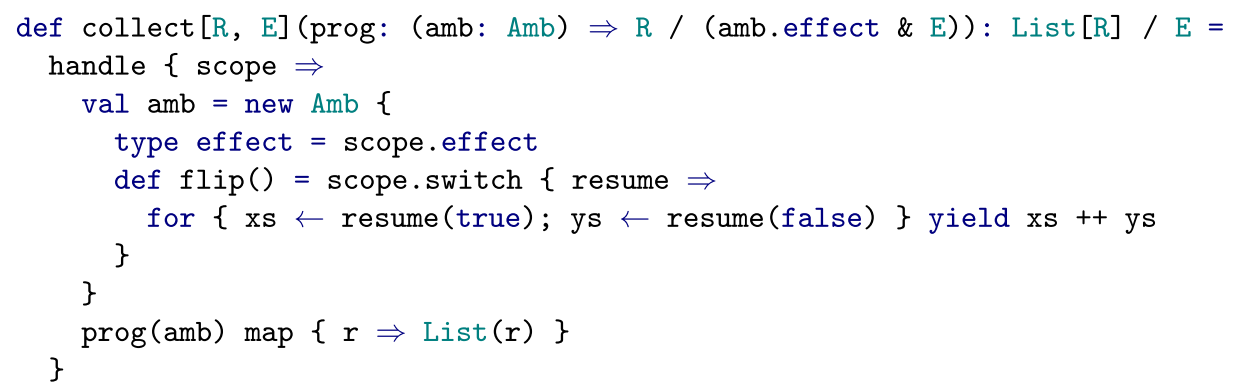

(c) Handler function for the ambiguity effect.

Fig. 2. Using Effekt to declare and handle exception and ambiguity effects.

\subsubsection{Capabilities are effect instances}

Consider the following effectful function which uses a capability for the exception effect ${ }^{2}$ :

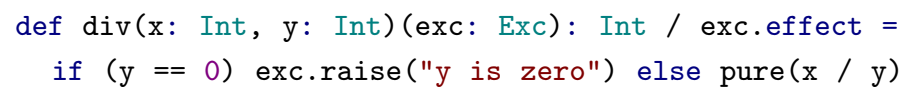

We read the type signature of div as "given an exception capability exc, div computes an integer using the capability exc". While mentioning exc twice in the type signature seems redundant, Effekt separates two concerns often conflated in existing effect languages.

2 To syntactically separate capabilities from other arguments, in the example, we curry the function definitions using multiple argument sections. 
Dynamic Effect Semantics. Passing exc as parameter gives the program (term-level) access to the methods of Exc, in this case the effect operation raise. Other languages with support for effect handlers perform a search at runtime to handle effect operations like raise. In contrast, using Effekt, we explicitly pass effect handlers in the form of capabilities as parameters.

Static Effect Semantics. When we use the effect operations of the exc capability to implement div, we have to mention the type member exc.effect in the effect type of div. We will go into the details of the effect system in Section 4 - for now it is enough to understand that we guarantee effect safety by tracking all unhandled effects in the type parameter Effects of Control, that is, the right-hand side of $R$ / E. Most of the time, the return type of effectful functions like div can be inferred.

\subsubsection{Effect handlers are implementations}

The effect signature Exc only specifies the available effect operations. To give them a concrete interpretation, we define a handler function with the following signature:

def maybe $[R, E](p r o g:($ exc: Exc $) \Rightarrow R /($ exc.effect \& E)): Option[R] / E

Handler functions fulfill two purposes. Firstly, they provide capabilities (i.e., exc) as arguments to the handled program. Secondly, handler functions remove the used effect (i.e., exc.effect) from the effect type. The type of prog is a path-dependent function type. The intersection type (i.e., exc.effect \& E) reflects that prog might use the exc capability passed to it, as well as other effects E. The handler function runs the program prog and effectively removes exc.effect so the final effect type is just $\mathrm{E}$. The handler is polymorphic in both - in the result type $\mathrm{R}$ of the handled program prog and in all other effects $\mathrm{E}$ that the program might use, and which are not handled by maybe.

Operationally, the handler function interprets the effectful program which would compute a result of type $\mathrm{R}$ (mnemonic for "result type") into a new semantic domain of type Option [R], the effect domain. As seen earlier, programs that raise exceptions will be handled to return None. Programs that do not raise an exception return Some (result).

Remark. We interchangeably use the terminology capability and handler instance. While "capability" puts a focus on the concept of entitling the holder to use an effect, "handler instance" highlights the fact that handlers are implementations of effect signatures.

\subsubsection{Handler implementations can shift the perspective}

Figure 2(b) uses the Effekt library to implement the handler function maybe. In Effekt, effect handlers can use other effects to implement effect operations. Often, handlers need to capture a delimited continuation. This is achieved using the built-in effect Scope, which declares the effect operation switch (Section 3). The Scope effect, in turn, can be handled with the built-in library function handle. It introduces a new capability, which we bind to scope in this example. Continuations captured by scope. switch will be delimited by this very call to handle. 
The anonymous handler instance exc extends the effect signature Exc and implements the method raise. It uses scope.switch to capture the current continuation. We communicate the use of scope on the type level by defining the type member effect to be scope.effect. Since we call handle at type Option $[R]$ / E, the method scope.switch has type:

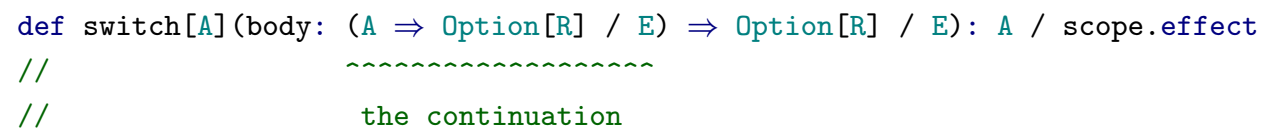

Calling scope. switch in our implementation of raise switches the current evaluation context: it transfers the control flow from the call site of raise to the respective (dynamically enclosing) call to handle. Conceptually, the body passed to switch is thus evaluated at the call to handle not at the call to raise. Calling switch also captures the current continuation and provides it as the argument (i.e., resume). The continuation allows us to transfer the control flow back to the original call site of raise. In this example, however, we discard the continuation and immediately return None. Operationally, the call to handle $\{\ldots\}$ will thus be replaced by pure(None), aborting the computation. The captured continuation resume corresponds to the context between the call to handle $\{$ scope $\Rightarrow \ldots\}$ and the call to scope.switch. While at runtime prog might arbitrarily install delimiters before calling raise, the connection between the two functions scope. switch and handle is statically scoped. We know that for all calls to exc.raise the captured continuation will be delimited by this lexically enclosing handle, even in the presence of duplicate instances of this delimiter as we discuss in Section 5.4.1.

\subsubsection{Return clauses}

Delimiting the scope with handle requires the result type of the passed program to match the effect domain of the handler - that is Option $[R]$ in our case. For this reason, we are mapping over the result of the program prog to wrap it in the constructor Some. In other languages like Koka, Eff, or Frank, this lifting of the result type into the effect domain is typically performed by return clauses. Using capability passing, return clauses are not required to be part of the user interface of handlers while maintaining the same expressive power (Section 5.5).

\subsection{Example: Ambiguity-resuming multiple times}

Our implementation of the exception effect discards the continuation of the program when it encounters a raise and immediately returns None. In contrast, the handler for ambiguity illustrates how to use the continuation to resume to the original call site. Figure 2(a) declares the effect signature of the Amb effect. The implementation of the handler function collect in Figure 2(c) handles the Amb effect. It changes the result type of the program from $R$ to the effect domain List $[R]$ :

def collect [R, E] (prog: (amb: Amb) $\Rightarrow R /$ (amb.effect \& E)): List [R] 
To implement the flip operation, we capture the continuation and call it with the result of the coin flip. Here, the type of the continuation is resume: Boolean $\Rightarrow$ List $[R] /$ E, so calling it with either true or false gives us a list of possible results List [R]. To explore all execution paths we call resume twice, once with true and once with false. Finally, we concatenate both lists with $x \mathrm{~s}++$ ys. Like before, we lift the result type $\mathrm{R}$ into the effect domain (this time List $[R]$ ) by wrapping the result in a singleton list. That is, if there is no call to flip, no ambiguity arises and the list contains only one result.

\subsection{The Effekt library}

In this section, we encountered the basic concepts of programming with effect handlers in Effekt. While all effectful computation happens in one monad (Control), programming with effect handlers encourages a modularization into three components: effect signatures, effectful programs, and effect handlers.

Effect signatures like Exc are interfaces containing methods marked as effectful with an abstract type member effect. This abstraction is very powerful — not only is the implementation of the method left abstract, but we also leave open which effects an implementation might use. In a concrete implementation, all effectful methods share the type member effect much like all methods of an object share the private state.

Effectful programs use effect operations by calling into explicitly passed capabilities. This has advantages, but also can be a burden due to its verbosity. Like we did in previous versions of Effekt for Scala (Brachthäuser \& Schuster, 2017), we could hide most of it using implicit parameters and implicit function types. However, for most parts of this paper, we refrain from doing so to reduce cognitive overhead and focus on the aspect of effect safety. Section 6.3.1 discusses the use of implicits in greater detail.

Effect handlers provide semantics to effect operations. We distinguish three different aspects of an effect handler. The handler function, like collect, is a higher-order function that provides an amb capability and removes the amb.effect from the effect type of the handled program. The handler implementation is a class implementing the effect signature. In our above example, the Amb interface is implemented by an anonymous inner class new Amb $\{\ldots\}$. The handler instance, like amb, is an instance of the handler implementation. Handler functions encapsulate three aspects of effect handling in one module:

1. the handler function uses handle to delimit the scope of the captured continuation;

2. it locally uses the fresh scope introduced by handle to implement the effect operations in terms of scope.switch - the effect operations close over scope and are thus the only way to capture the continuation;

3. it finally lifts the return type of the handled function $\mathrm{R}$ into the effect domain which makes it the answer type of the delimiter handle.

Grouping these aspects of effect handling in one module, it is possible to locally reason about type safety. The implementation of raise is only safe because we statically know that the answer type expected at handle is Option $[R]$. Likewise, in collect, we know that the answer type in the body of scope.switch is List [R]. This allows us to safely concatenate the results of the two calls of the continuation resume. 
The remainder of this paper iterates the running example of this section and introduces all mentioned types and library functions in three steps: Section 3 gives an overview over the underlying implementation of delimited control. Section 4 then establishes effect safety for this implementation of delimited control and introduces the abstraction of effect signatures. Finally, Section 6 explores newly gained extensibility by refactoring the handler implementations of maybe and collect into reusable and extensible traits.

\section{Answer-type safe effect handlers}

Effekt implements effect handlers in terms of a monad for delimited control. In previous sections, we used a monad Control [+Result, -Effects] that is both answer-type safe and effect-safe. To focus on the operational semantics of delimited control and to illustrate problems of effect safety, in this section, we start with a simpler variant Control [+Result] which has the same operational semantics is answer-type safe, but not effect-safe. It roughly corresponds to the one presented in earlier work (Brachthäuser \& Schuster, 2017). In Section 4, we show how to achieve effect safety.

\subsection{Structured programming with delimited continuations}

For multiple decades, control operators like call/cc have been used to program with control effects (Friedman et al., 1984). Recently, in disguise, control effects have found their way into mainstream programming languages in the form of specialized solutions such as async/await, fibers, coroutines, generators, and others.

Also recently, the programming languages research community found new interest in control effects in the form of algebraic effects (Plotkin \& Power, 2003) and their extension with handlers (Plotkin \& Pretnar, 2009, 2013). Effect handlers occupy a sweet spot between the general control operators (such as call/cc) and specialized programming language features (such as async/await). Like general control operators, effect handlers are very powerful and can express many of the above language features as user-defined libraries (Dolan et al., 2015, 2017; Leijen, 2017b). However, unlike general control operators, effect handlers also encourage modularity. We believe the regained interest comes from four important improvements over control operators like call/cc:

1. generalizing from undelimited to delimited continuations

2. generalizing from one control operator to a family of control operators

3. establishing answer-type safety of control operators

4. establishing effect safety of control operators

From an engineer's perspective, each of these improvements helps to write programs in a modular way making them easier to extend and making it easier to reason about parts of a program in isolation.

The connection between effect handlers and delimited control is not accidental. It has been established practically (Kiselyov \& Sivaramakrishnan, 2018) as well as theoretically (Forster et al., 2017; Piróg et al., 2019) that certain forms of delimited continuations can express certain forms of algebraic effect handlers. Similarly, in the literature, effect handlers are sometimes introduced as a structured way to programming with delimited continuations (Kammar et al., 2013; Leijen, 2017c). 


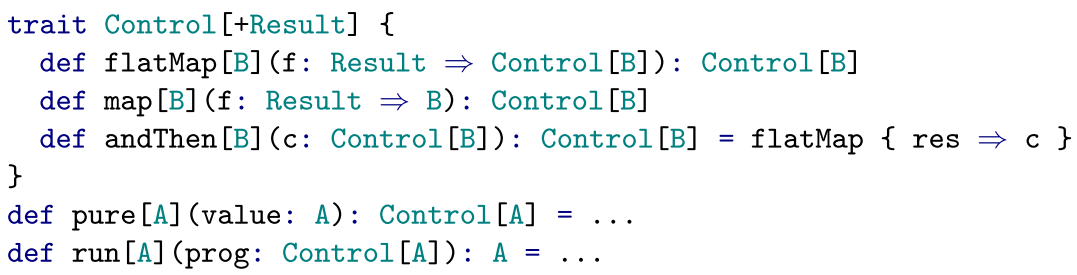

(a) The monad Control for programming with delimited control.

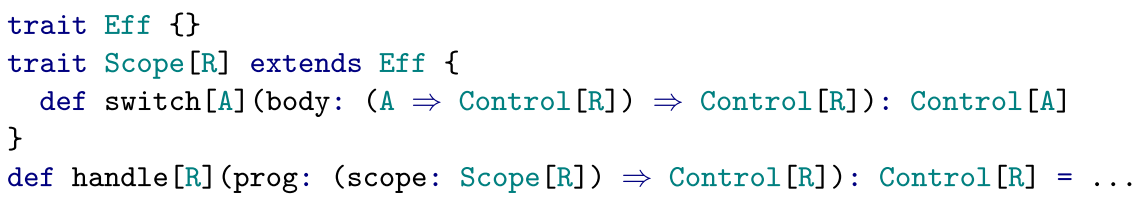

(b) The Scope effect with effect operation switch, and built-in handler function handle.

Fig. 3. The API of Effekt without effect typing.

"Effect handlers are to delimited continuations as structured programming is to goto"attributed to Andrej Bauer by Kammar et al. (2013).

In the design of the Effekt interface (presented in Figure 3), we take this quote literally. In particular, there are two ways to view the monad Control.

An Embedding of Delimited Control. One can view Control as an embedding of a language with (delimited) control effects into Scala. The library function handle is a delimiter for the control effects in the provided program prog. It also introduces a fresh Scope that can be thought of as a prompt marker (Sitaram \& Felleisen, 1990; Gunter et al., 1995) labeling the corresponding call to handle. The method switch is a control operator. It captures (and removes) the current continuation up to (and including) the corresponding handle. It then passes the continuation to the provided body. Taking this point of view, programming with Control as presented in the introduction is "just" structured programming with delimited continuations.

Delimited Control as Built-in Effect. For the purpose of this paper, however, we want to take an alternative point of view. Staying in the conceptual framework of effect handlers, we think of switch as an effect operation, defined in the effect signature Scope. The function handle is a handler function for the Scope effect. Effect handlers can be defined in terms of other effects, including the Scope effect. The only difference between the Scope effect and user-defined effects like Amb or Exc is that the handler function handle is built into the Control monad. This point of view emphasizes programming with effects and handlers. The potential use of the scope effect to capture the continuation is an implementation detail of the respective user-defined handler.

\subsection{The Control monad-Delimiting continuations}

Figure 3(a) defines the interface of our monad for delimited control. The implementation of our monad Control [+Result] is based on the monad for multi-prompt delimited control 
by Dybvig et al. (2007). We specialize both, syntax and semantics, to better fit effect handlers. As usual, we embed a pure value into the monad with pure and we sequence effectful computations with flatMap. This enables us to write effectful programs in an imperative style via Scala's for-comprehensions. Using run [A], we execute a program with control effects that computes a value of type A. The type parameter Result of our monad is marked as covariant (i.e., +Result) to enable correct subtyping.

\subsubsection{Capturing delimited continuations with Scope. switch}

Besides being a monad, Control enables the implementation of one particular built-in effect: Scope. Figure 3(b) defines the Scope effect and its corresponding built-in handler handle. The effect operation switch captures the continuation and binds it to resume. It is important to emphasize that the continuation is delimited. Such a delimited continuation (Felleisen, 1988) is also referred to in literature as subcontinuation (Hieb et al., 1994) or partial continuation (Johnson \& Duggan, 1988). Since their introduction by Felleisen, many variants of delimited control operators have been discovered-maybe the most prominent example being shift / reset (Danvy \& Filinski, 1992). Using shift / reset, we can express the following example:

$\operatorname{print}(\operatorname{reset}\{(1+\operatorname{shift}\{k \Rightarrow k(3)+k(4)\}) * 2\})$

The continuation captured by shift (highlighted in gray) corresponds to the evaluation context $(1+\square) * 2$. Its extent is delimited by reset and thus does not include the call to print. The example will print the number $((1+3) * 2)+((1+4) * 2)=18$. Importantly, since it is delimited, calling the continuation $k(3)$ does return with the value 8 .

\section{Example 1}

We can translate the above example to Scala using the Control monad and the Scope effect:

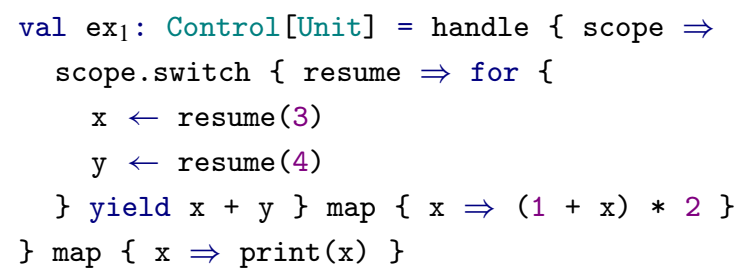

Like above, running run $\left\{\operatorname{ex}_{1}\right\}$ will print the number 18. Adjusting the example illustrates the choice of terminology for scope. switch.

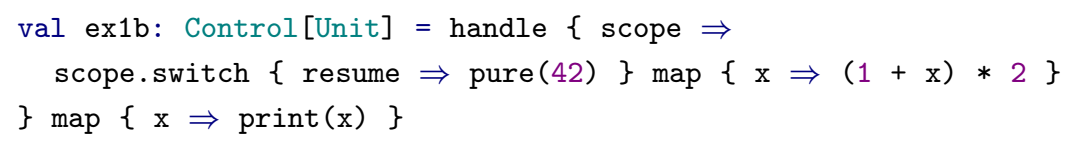

The call to switch changes (i.e., "switches") the context to the handle call that introduced the scope. The body passed to switch is evaluated in the context print $(\square)$. Returning from the body $i$ returning from handle and running run $\{$ ex1b $\}$ prints 42 . 


\subsubsection{Multiple scopes and families of control operators}

We will now see how to use the Control monad to program with multiple scopes (Sitaram \& Felleisen, 1990; Gunter et al., 1995). Every call to handle introduces a fresh scope that corresponds to a prompt marker (Sitaram \& Felleisen, 1990). This gives rise to a dynamic number of control operators scope.switch, one for each call to handle. The following example illustrates the use of multiple delimiters.

\section{Example 2}

We use handle twice, introducing two different scopes $s_{1}$ and $s_{2}$.

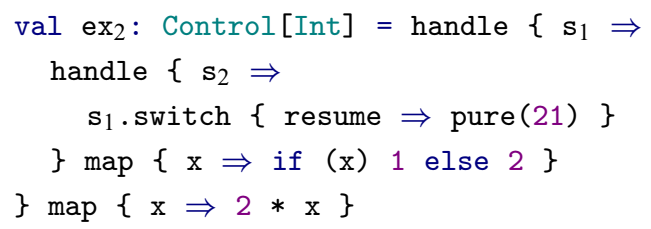

The captured continuation resume contains the program segment delimited by scope $\mathrm{s}_{1}$, that is, the evaluation context handle $\left\{s_{1} \Rightarrow\right.$ if (handle $\left\{s_{2} \Rightarrow \square\right\}$ ) 1 else 2$\}$. Here, the body of switch discards the continuation and returns 21 . Hence, $\operatorname{run}\left\{\operatorname{ex}_{2}\right\}$ gives 42 .

\subsubsection{Answer-type safety}

Operationally, scope.switch $\{\mathrm{k} \Rightarrow$ PROG $\}$ replaces the corresponding call to handle by the body PROG. The return type of the body has to match the answer type at the handle. To guarantee this, we follow Gunter et al. (1995) and parametrize the Scope effect by the answer-type R (Figure 3(b)). In Example 2, the two scope capabilities thus have types $\mathrm{s}_{1}$ : Scope [Int] and $\mathrm{s}_{2}$ : Scope [Boolean]. The type of handle [R] in Figure 3(b) requires three types to be $R$ : the answer type of the created scope (i.e., Scope $[R]$ ), the result of the given program (i.e., Control $[R]$ ), and the return type of handle. Similarly, the type of switch uses the answer type of the given scope and requires that (a) the return type of the continuation and (b) the return type of the given body agree with (c) the answer type expected at the handle that introduced this scope. Answer-type safety is especially important for multiple nested scopes. Each call to handle might introduce a scope with a different answer type. Switching to a handler with the wrong type should be statically rejected. For instance, switching to $s_{2}$ instead of $s_{1}$ would render Example 2 type-incorrect. It would require the body of switch to return a computation of type Boolean, not Int.

\section{Effect-safe effect handlers}

In the previous section, we have seen a version of Control that has a type parameter Result. Also indexing Scope with Result, we statically track answer types and guarantee that capturing and calling the continuation is type-safe. However, this version of Control is not effect-safe: capabilities can leave the scope of the handler function that introduced them, that is, the handler region. Using a Scope capability outside of the handler 
region leads to a runtime error. We illustrate two ways for capabilities to leave the handler region.

Leaving the Handler Region by Returning. We can leave the handler region by returning from it. In this case, it is possible to leak the Scope capability either through references

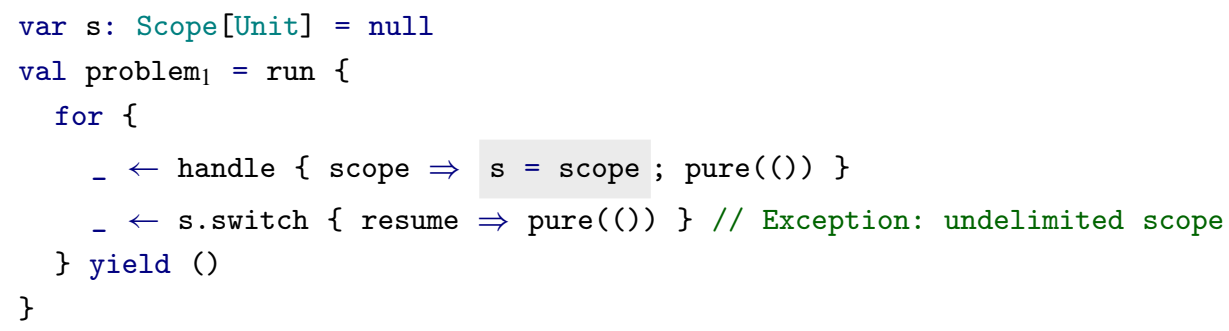

or simply by returning it as result:

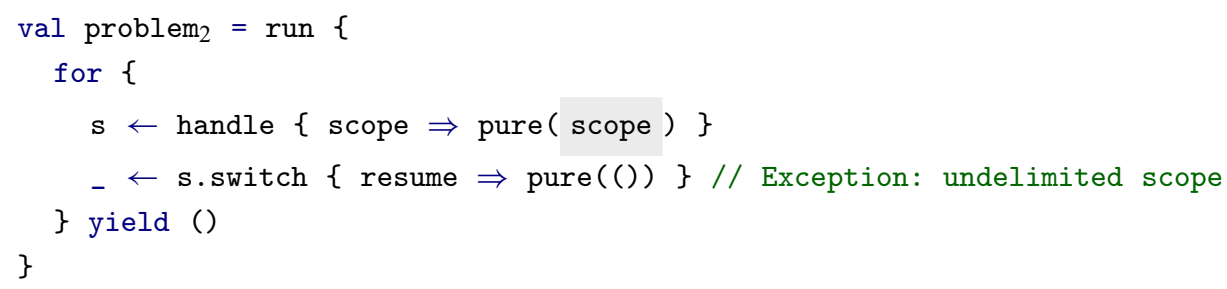

As also observed by Osvald et al. (2016), both sources of leakage can occur indirectly through functions or objects that close over the capability. Capabilities might even leave the scope of the enclosing run to then be used in the scope of a different run. Dybvig et al. (2007) use rank-2 types to prevent this particular source of runtime errors, but leave others to future work.

Leaving the Handler Region by Scope Switching. We can also leave the scope of handle by means of control effects, that is, by switching the scope.

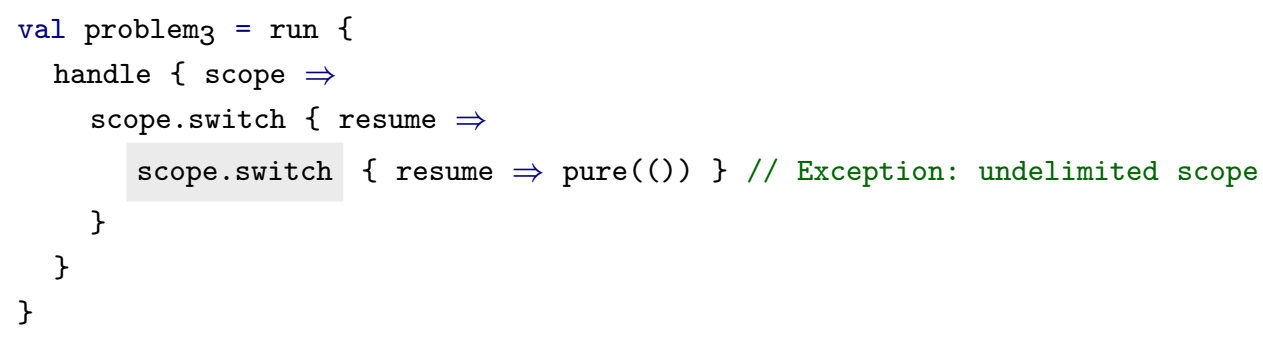

Since switching the scope removes the enclosing handle, switching a second time inside of the body of switch results in a runtime error. The evaluation context of the second switch is run $\{\square\}$ and the captured continuation is not delimited anymore. Danvy \& Filinski (1990) operationally prevent this kind of runtime error by leaving the outer delimiter behind. That is, the evaluation context would correspond to run $\{$ handle $\{$ scope $\Rightarrow \square\}$ \}. However, in 
the setting of multiple prompts/scopes this is not sufficient. The delimiter can be removed by switching to a different scope:

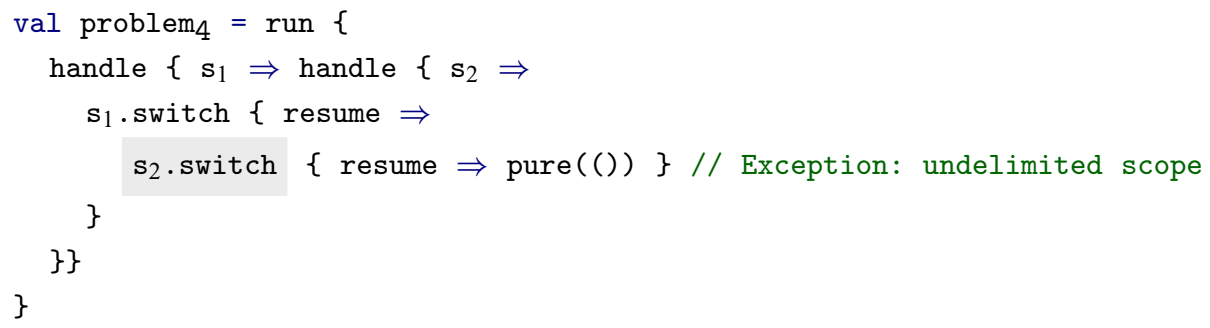

\subsection{Establishing effect safety}

We now introduce our implementation of an effect system that rules out the above four problematic programs, prevents the use of escaped capabilities, and guarantees effect safety. The underlying problem that our effect system solves is a general one: we need to restrict the lifetime of a resource (capabilities in our case) to a certain dynamic region (the call to handle in our case). This problem occurs in the domain of regionbased resource management (Kiselyov \& Shan, 2008), object capabilities (Haller \& Loiko, 2016), delimited control (Dybvig et al., 2007), scope safety in type-safe metaprogramming (Parreaux et al., 2017), as well as with prompt-based implementations of effect handlers (Brachthäuser \& Schuster, 2017). Our effect system is inspired by the $\lambda_{\sqrt{\Omega}}$ calculus (Zhang \& Myers, 2019), which uses dependent types to track the set of used labels (i.e., capabilities) in the effect type. In Section 5, we discuss some properties of our effect system embedding, but leave formal proofs of safety and soundness to future work. For a better comparison, Appendix A gives a more immediate embedding of $\lambda_{\Omega 仓}$ into Scala.

\subsection{Tracking capabilities}

Following Zhang \& Myers (2019), our effect system builds on the idea of tracking the set of capabilities used by a program in the type of the program. To enable tracking of effects, Figure 4 thus defines our final version of Control with a second-type parameter Effects. We represent capabilities (i.e., instances of type Eff) on the type level by their abstract type member effect and we use Scala's intersection types to describe a set of capabilities.

As an example, assuming capabilities $c_{1}, c_{2}$, and $c_{3}$ we write the type of the effectful program that uses $c_{1}$ and $c_{2}$ to compute an integer as:

val $\operatorname{prog}_{1}$ : Control[Int, $c_{1}$.effect \& $c_{2}$.effect]

Here, $c_{1}$. effect is the abstract type member of the capability and $c_{1}$. effect \& $c_{2}$. effect is an intersection type. The intersection might be uninhabited, but this is irrelevant for our use case. We only use the intersection type as a phantom type to track used effects.

To support effect subtyping, the type parameter Effects of Control is marked as contravariant (i.e., -Effects).

val $\operatorname{prog}_{2}$ : Control[Int, $c_{1}$. effect \& $c_{2}$. effect \& $c_{3}$.effect] $=\operatorname{prog}_{1}$ 
type Pure = Any

type $/[+R,-E]=\operatorname{Control}[R, E]$

(a) Type aliases Pure to describe the empty set of effects and / for infix notation.

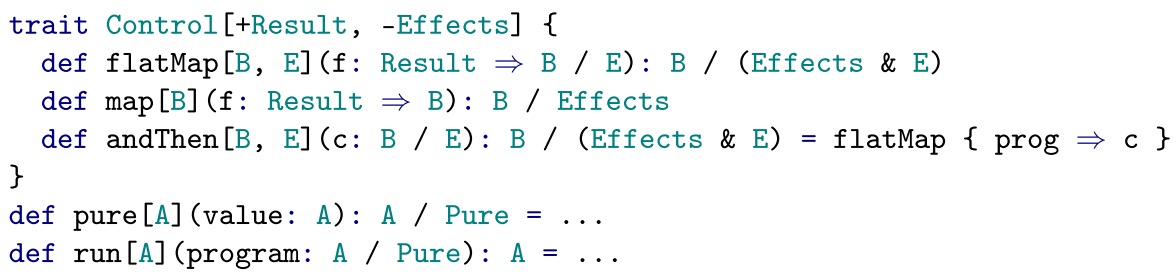

(b) The monad Control for programming with delimited control.

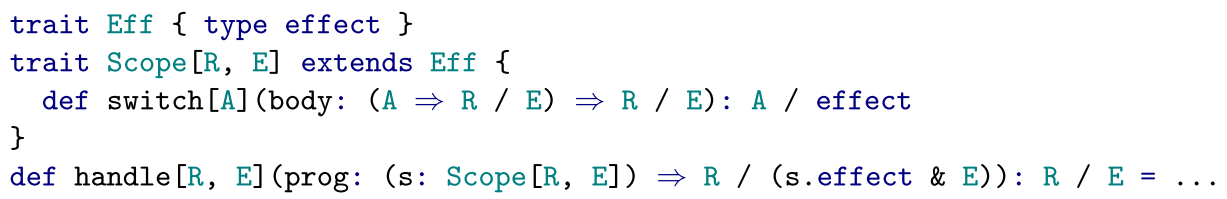

(c) The Scope effect with effect operation switch, and built-in handler function handle.

Fig. 4. The API of Effekt with effect typing.

The above assignment is type correct, since we have by subtyping:

$c_{1}$.effect \& $c_{2}$.effect \& $c_{3}$.effect $<: c_{1}$.effect \& $c_{2}$.effect

We model the syntactically empty intersection of capability types by defining the type alias type Pure = Any, where Any is the top of the Scala subtyping lattice. Pure programs have an effect type Pure since they do not use any capabilities. By contravariance, they are a subtype of effectful programs that do contain capability types in the intersection. It is important to point out that the purity only refers to delimited control. Programs with a pure effect row still can use side effects like writing to files or accessing the network.

Every use of a Scope capability, for example, scope.switch, taints the effect type of its result. The return type A / effect of switch (Figure 4) indicates the use of switch on this capability on the type level. The type of run asserts that only pure programs without control effects can be executed. That is, all capabilities have to be removed from the set of effects and it has to be Pure. To guarantee safety, we have to make sure that the only way to remove an effect from the intersection is by delimiting the program with handle:

$$
\text { def handle }[R, E] \text { (prog: (s: Scope }[R, E]) \Rightarrow R /(\text { s.effect \& E)): R / E }
$$

Here, prog has a dependent function type: the return type is (path)-dependent on its value parameter s. Importantly, in our implementation, we leave the type member effect of $s$ abstract. Different calls to handle lead to different types s.effect that cannot be unified by the type checker. Hence, only the call to handle that introduced a scope capability can remove its very own abstract effect type from the set of effects. This excludes problematic programs like problem 1 and problem $\mathrm{em}_{2}$ from above.

Our approach to achieve effect safety is similar to how rank-2 types can be used to enable type-safe monadic regions in Haskell (Launchbury \& Sabry, 1997; Moggi \& Sabry, 2001; 
Kiselyov \& Shan, 2008). Using path-dependent types, we move the universal quantification from the type level to the term level. Since rank-2 types are not well supported in Scala, using abstract type members also improves ergonomics and type inference.

Remark. In Scala, two (path-dependent) types are equal if and only if their prefix paths are stable and they can be unified (Odersky \& Zenger, 2005b). Informally, a path is stable if it does not contain a mutable component. This way we prevent leakage via mutable references as in problem .

\subsection{From answer-type safety to effect safety}

In the previous variant of Control, scopes carried the answer-type $R$ to ensure that using control effects is type-safe. Crucially, to establish effect safety and prevent programs like problem 4 from type checking, the type scope (Figure 4) now has an additional type parameter E.

trait Scope [R, E]

With this change, scope capabilities now track both the expected return type $R$ and the set of capabilities E available at the corresponding handler. The type Scope $[R, E]$ can conceptually be understood as type Scope $[R / E]$. However, we track the two aspects in separate type parameters to improve type inference.

Intuitively, the body of a switch is evaluated at the position of the corresponding call to handle. This is also reflected in the type of the body:

body: $(A \Rightarrow R / E) \Rightarrow R / E$

Both, the answer-type $R$ and the effects $E$ have to match the corresponding handle $[R, E]$. Thus, capability-passing style is not only essential for operationally delimiting control effects but also necessary to carry both the expected answer type as well as the available effects from the definition site handle $\{$ scope $\Rightarrow \ldots\}$ down to the use site scope.switch.

Since the body of switch has to return R / E, it cannot shift to the same scope again (as in problem 3 ). This would require a type of $R /$ (scope.effect \& E). The problematic program problem 4 is ruled out too: $s_{1}$ has type Scope [Int, Pure] and thus the body of the first switch needs to be pure and cannot use $s_{2}$.

\section{Example 1 -Effect typed}

We are now ready to revisit the examples from the previous section and assign effect types. On the term level, the example is the same, but its typing is more precise:

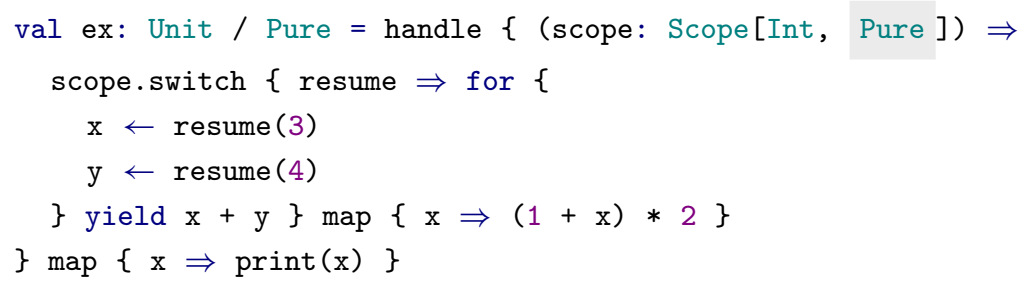


The effect type of ex shows that after handling there are no more undelimited control effects left and we can safely run the program.

\section{Example 2 -Effect typed}

The second example illustrates how each handle removes its corresponding scope capability from the effect type.

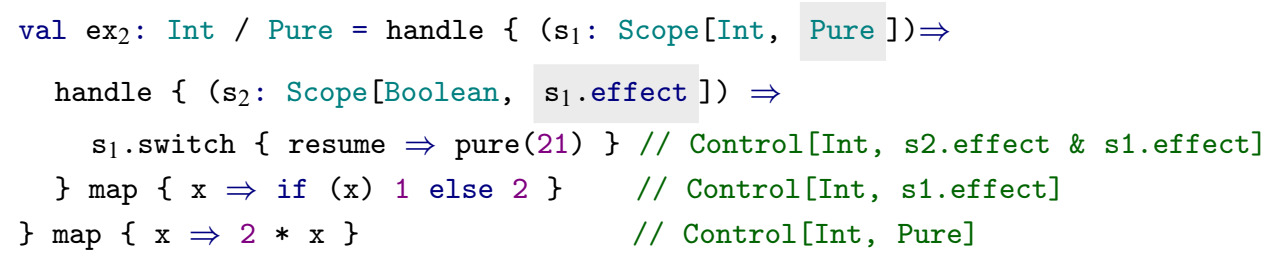

The body of the second handle has type Control[Int, $s_{1}$.effect \& $s_{2}$.effect]. By effect subtyping, we can still use $s_{1}$. switch $\{\ldots\}$, which has type Control [Int, $s_{1}$.effect].

The example also highlights an important aspect of our effect-safe control operator: scope capabilities track the available effects at the definition site, not the use site. For instance, the type of $s_{2}$ informs us that within a body of $s_{2}$. switch $\{\ldots\}$, the $s_{1}$. effect can be used. In contrast, the return type of $s_{2}$. switch in this example would only mention $s_{2}$.effect, not $s_{1}$.effect. From the point of view of effect handlers, the potential usage of $s_{1}$. effect in the body of $s_{2}$. switch $\{\ldots\}$ is an implementation detail that is encapsulated at the definition site. It does not leak to the use site.

\section{Coin flipping example - Effect typed}

The handler function maybe from Section 2 implements a user-defined effect Exc in terms of another effect Scope. Let us recall the effect signature of Exc

trait Exc extends Eff \{ def raise(msg: String): Nothing / effect \}

and the (type annotated) maybe handler function:

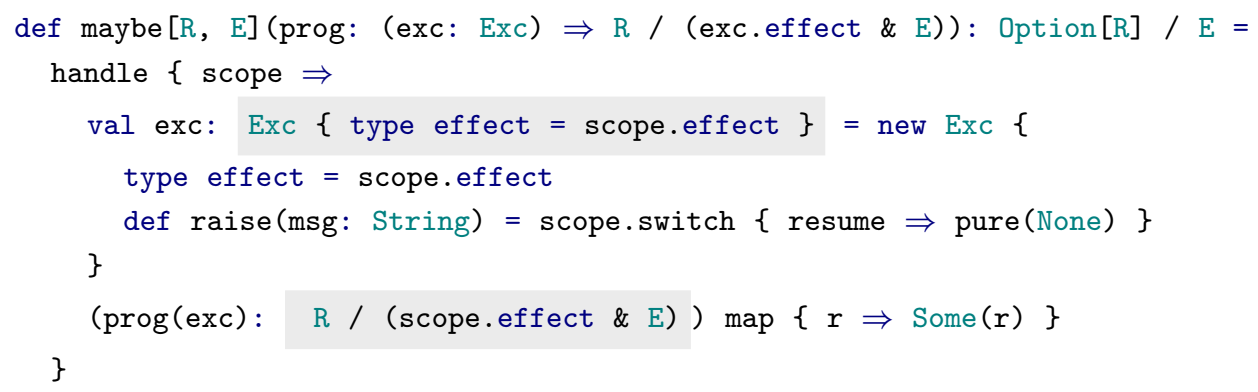

The implementation exc of Exc uses the scope capability. The type of exc is therefore refined from Exc to Exc $\{$ type effect = scope.effect $\}$. This allows the type checker to locally unify exc.effect and scope.effect. It is also necessary for handle to remove the scope.effect component of the effect type, resulting in the return type Option $[R]$ / E. Since the type refinement is local, the use of the capability scope in the 
implementation of exc and the type equivalence between exc.effect and scope.effect remains unknown to the handled program prog. This implementation detail is encapsulated in the maybe handler function. Similarly, as we will see in Section 6, also the usage of effects other than scope can be encapsulated.

Having introduced the effect system, we are ready to type- and effect-check the example program from the introduction:

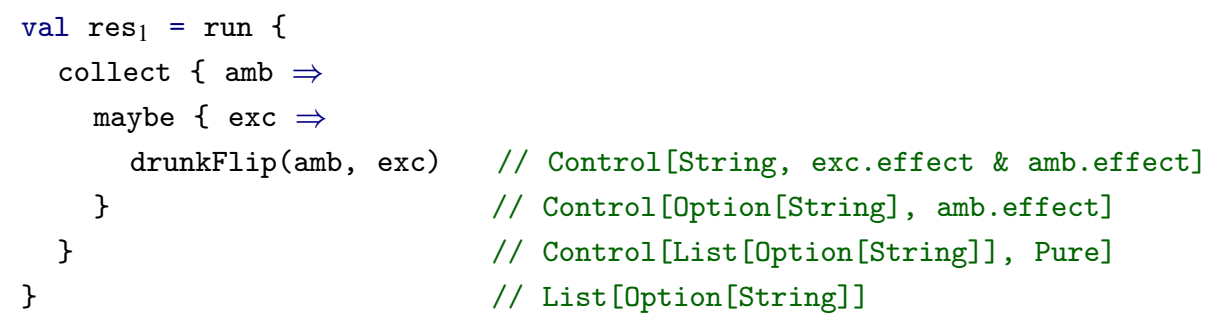

Each handler function removes an effect and at the same time changes the effect domain.

\subsection{Effect-safe ambient state}

Many practical handler implementations require some form of state. Our host language Scala already supports mutable state and effect handlers can readily use it. However, combining mutable state and delimited control can interact in unforeseen ways (Kiselyov et al., 2006; Leijen, 2018). This is illustrated by the example in Figure 5(a). In the program on the left, we introduce a local mutable variable $\mathrm{x}$. We only modify it if the flip operation returns true. Still, running the example outputs List $(2,2)$. Surprisingly, the change to $x$ is also visible in the branch where flip returns false. Built-in mutable state is global and does not backtrack across different resumptions.

For some effect handlers, we want local, backtrackable state. Running the example program should return List $(2,0)$, backtracking the local state when resuming the second time. Leijen (2017c) calls this form of state "ambient". There are multiple ways to achieve this desired behavior. One way is to define a state effect in terms of the Scope effect. For effect handlers, this technique has been presented by Kammar et al. (2013), and for delimited control by Kiselyov et al. (2006). Another way would be to offer a generalized form of effect handlers that support local state. For example, Koka (Leijen, 2017c) supports "parametrized handlers" which manually perform state-passing.

\subsubsection{The State effect}

For our design of Effekt, we explore a third approach and offer a built-in State effect that exhibits the correct backtracking behavior in combination with the Scope effect. Figure 5(b) defines the interface of the effect signature state and the corresponding built-in handler region. The use of the state effect is illustrated in the right column of Figure 5(a). Given a capability state, we create a new field $x$ with val $x=$ state.Field (...). To read and update the state, we use the effect operations get and put on the field. Our types make sure that we can only access a field within the region of the state capability that we used to create the field. 
in this section, we discuss some properties of our embedded effect system and explain under which assumptions we believe them to hold.

\subsection{Effect safety}

Effect safety is the absence of runtime errors, caused by capabilities being used outside of their defining handlers. Assuming a sound subset of Scala, such as the pDot calculus (Rapoport \& Lhoták, 2019), we are confident that our effect system establishes effect safety-though we do not give formal proofs. Adding mutable variables and fields to the calculus should also not affect our effect system, which relies on stable, that is, immutable paths. The same also holds for native exceptions, though our library as presented in this paper is not prepared to interact with native exceptions. A formal treatment is left to future work. In our experience, adding effect types to existing advanced case studies (such as the Scheduler handler in Section 7) helped us to discover a few subtle bugs. The effect system also guided us in the design of the interface for ambient state as presented in Section 4.4.

\subsection{Effect subtyping}

By marking the set of capabilities in Control as contravariant, we use Scala's support for subtyping of intersection types to express effect subtyping. For example, a program only using the amb effect is a subtype of a program using amb and exc:

Control [R, amb.effect] <: Control [R, amb.effect \& exc.effect]

As explained in Section 4, this is the case since we have

amb.effect \& exc.effect <: amb.effect

Reusing Scala's subtyping for effect subtyping is an important advantage over effect systems that encode effect rows using type-level lists. In those systems, effect subtyping typically has to be implemented manually by performing type-level computation (Kiselyov \& Ishii, 2015). In contrast, using intersection types to express the set of effects integrates well with other Scala features like variance annotations and type bounds. Type inference for monotonically growing intersection types is well supported, and in consequence most return types of effectful functions (like drunkFlip) and handlers (like maybe) can be omitted.

\subsection{Effect polymorphism}

Since effect types are Scala types, we can also reuse Scala's support for type polymorphism to express effect polymorphic functions. One example of an effect polymorphic, higherorder function is

$\operatorname{def} \operatorname{mapM}[A, B, E]($ lst: List $[A], f: A \Rightarrow B / E):$ List $[B] / E$

The function mapM is effect polymorphic in the effects $E$ used by function $f$. The return type of mapM indicates that it potentially calls $f$ in its implementation and so has the same 
effects as $f$. The effects $E$ still need to be handled by the caller of mapM. Handler functions like collect and maybe (Section 2) are other examples for effect polymorphic functions. Type inference for calling higher-order functions (like mapM) that do not alter the set of effects is well supported. In contrast, nesting multiple handler applications (like collect and maybe) often requires users to explicitly list the remaining effects by provide typing annotations.

\subsection{Effect parametricity}

The example function mapM is polymorphic in the effects E. Following Zhang \& Myers (2019), we claim that it should not be possible for the implementation of mapM to (accidentally) handle any concrete effect in $\mathrm{E}$; no matter what $\mathrm{E}$ will be instantiated to at the call site. That is, in the following user program, we should be able to determine statically that flip is handled by collect. No implementation of mapM should be able to violate this assumption.

collect $\{\mathrm{amb} \Rightarrow \operatorname{mapM}(\operatorname{List}(1,2,3), \mathrm{n} \Rightarrow \operatorname{amb} . f l i p())\}$

We refer to this property as effect parametricity. It has also been called abstraction safety in the literature (Zhang \& Myers, 2019). Generally speaking, given a type, parametricity allows us to infer properties of the runtime behavior. In the case of effect parametricity, we want to guarantee the absence of accidental handling. But does effect parametricity hold for our implementation of Effekt? The answer is more subtle than in most other implementations of effects and handlers because Effekt is based on capability passing. We need to distinguish two different aspects of accidental handling:

Implementation Parametricity. Accidental handling of effects can occur in languages without static effect systems like Eff (Bauer \& Pretnar, 2015) and Multicore OCaml (Dolan et al., 2014). Those languages dynamically search handlers at runtime. In those systems, mapM could (accidentally or purposefully) handle Amb and, for example, change the semantics of the flip operation to always return true. Previous presentations of Effekt (Brachthäuser \& Schuster, 2017; Brachthäuser et al., 2018) did not have an effect system. Still, they already support this aspect of effect parametricity. Just like presented in this paper, user programs are written in capability-passing style. Capabilities are passed down to their use site and not looked up at runtime. In our example, the function passed to mapM closes over the capability amb, which fixes the implementation of $f l i p$.

Scope Parametricity. Because Effekt uses an implementation of multi-prompt delimited continuations under the hood, there is a second aspect of effect parametricity to consider. Looking at the example call to mapM again, we would also like to be sure that the continuation captured by flip will always be delimited by the corresponding call to collect and nowhere else. Because mapM does not know about amb, it should not be possible for mapM to install a scope delimiter for amb. In Effekt, we can construct examples that violate this property as we will see next. 


\subsubsection{Capturing the scope delimiter by leaking the continuation}

Every call to handle creates a fresh scope. Surprisingly, it is possible to delimit a program given an existing scope, even if we do not offer this in the API of Effekt. ${ }^{3}$ The following example illustrates (accidental) delimiting of continuations.

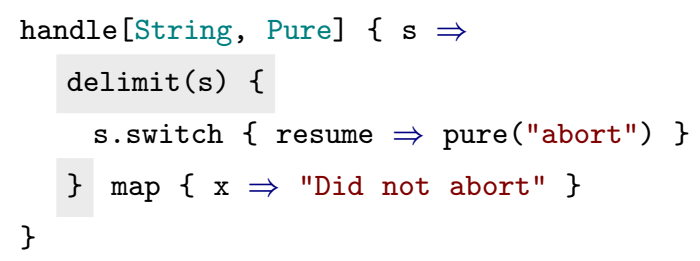

We use handle to delimit a program and create a new scope s. Within the delimiter, we then use s.switch to abort the current computation and return the string "abort" as the overall result. We now want to statically know that the continuation captured by s.switch is delimited by the call to handle that created $s$, independent of the implementation of delimit. In particular, we might think that running the program will always return the string "abort", given that delimit forces the computation passed to it. In Effekt, this does not hold. Maybe surprisingly, we can write a function delimit, such that the example program returns the string "Did not abort" instead of aborting to the outer scope.

Installing Delimiters by Resuming. The continuation captured by s. switch contains the delimiter for $\mathrm{s}$ and calling the continuation reinstalls the delimiter. This fact is not represented in the type of resume. It only mentions the effects it uses not the ones it delimits. Using this insight, we can define delimit:

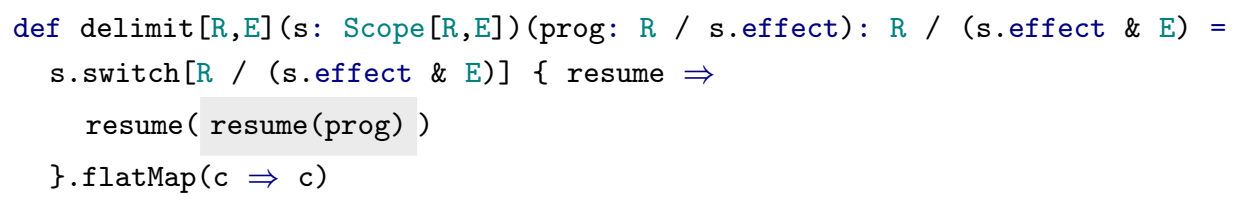

The implementation of delimit captures the continuation resume, which contains the delimiter for scope s. The call to s.switch returns a computation that will call the continuation on the given program. We force this computation outside of the call to s.switch with flatmap $(c \Rightarrow c)$. This effectively duplicates the captured context and the delimiter for $\mathrm{s}$, as illustrated by the following reduction:

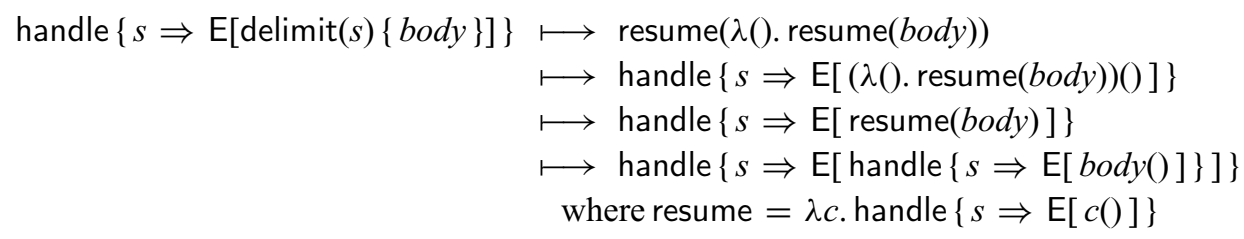

Here, we use $E$ to denote the evaluation context between the call to delimit and the corresponding handler for s (Wright \& Felleisen, 1994). Evaluating the call to delimit reifies the context and binds it to resume. Forcing the passed continuation (e.g., $c())$ is part of this captured context. Now, calling the continuation reinstalls the evaluation context between

3 We are grateful to an anonymous reviewer, who pointed out this source of violating parametricity. 
the call to delimit and (including) the original call to handle. Calling it again, after the context has already been restored, duplicates the context. If body now captures and discards the continuation, only the innermost copy of the context handle $\{s \Rightarrow \mathrm{E}[\square]\}$ is removed. Hence, our example returns "Did not abort".

Importantly, the captured context E can contain arbitrary other handler frames for scopes $s \neq s^{\prime}$. That is, all other delimiters in the evaluation context $\mathrm{E}$ are duplicated, even though the method delimit neither has access to them nor mentions them in its type signature.

\subsubsection{Discussion}

As can be seen from the example, violating scope parametricity requires some careful engineering. While it is necessary for the continuation to leave the effect operation that captured it, this is not sufficient. It is important to note that just calling the continuation twice (e.g., resume () ; resume ()) does not cause the capturing behavior. When the continuation is called for the second time, the delimiters reinstalled by the first call have already been removed. The same holds for executing code after calling a continuation (e.g., resume ()$; f())$. Effects in $f$ are not captured by the delimiters in resume. To observe accidental capture, the executed code has to be part of the continuation itself. In our example, we use a higher-order function and pass the code to be executed to the continuation.

In our experience in working with the library, we did not find the lost scope parametricity to be a problem in practice. To instantiate multiple copies of the continuation required us to use higher-order, effect polymorphic effect operations. Other effect handler implementations come with different linguistic restrictions, ruling out this particular capturing scenario. For example, Koka and the $\lambda_{\Omega \text { 仓 }}$ calculus do not support effect polymorphic effect operations, which makes it difficult to express delimit. Scala Effekt is a practical implementation, embedded in Scala. Using Scala's type polymorphism to model effect polymorphism, effect operations can naturally also make use of this feature. It is not clear to us which restrictions are desirable and practically enforceable without ruling out useful programs (like the ones in the following section).

Finally, we are not aware of any effect handler language, where the type of the continuation reflects the effects it handles. Depending on the other features of the particular language, it thus might still be possible to construct a scenario similar to the one described above. We leave it to future work to further investigate the problem and explore solutions, both for stand-alone languages, as well as for embeddings like Effekt.

\subsection{Effect encapsulation}

Another property, effect encapsulation, is related to effect parametricity, and violations of it can be observed in languages featuring an ML-like type system with row polymorphism for effect types like Koka and Frank (Lindley, 2018; Leijen, 2018). The following program is adapted from Leijen (2018) and written in the Koka language. It shows how an effect used by $f_{1}$ leaks into its type-it is not encapsulated.

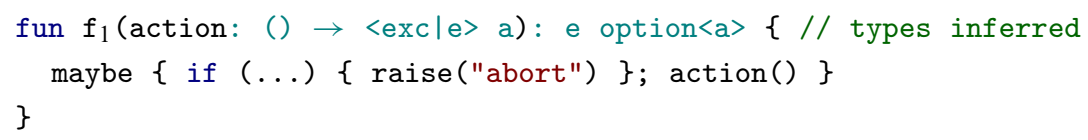


Here, $f_{1}$ is a higher-order function that takes an effectful function action as its argument. The function $f_{1}$ uses exceptions in its implementation but locally handles them with the maybe handler. This implementation detail leaks as part of the inferred type which states the fact that exc effects of action will be handled by $f_{1}$. Koka implements effect subtyping via row polymorphism so the effect row of action() is unified with those of other statements handled by maybe.

\subsubsection{Manually encapsulating effects}

Operationally, Koka will handle any exception effect used in action with the maybe handler in $f_{1}$. We cannot hide this fact by annotating the parameter action of $f_{1}$ with the type ()$\rightarrow$ e a, which does not type-check. However, if we do not want any exceptions thrown by action to be handled by maybe, languages like Koka and Frank offer some form of manual lifting operation (Biernacki et al., 2017; Convent et al., 2019).

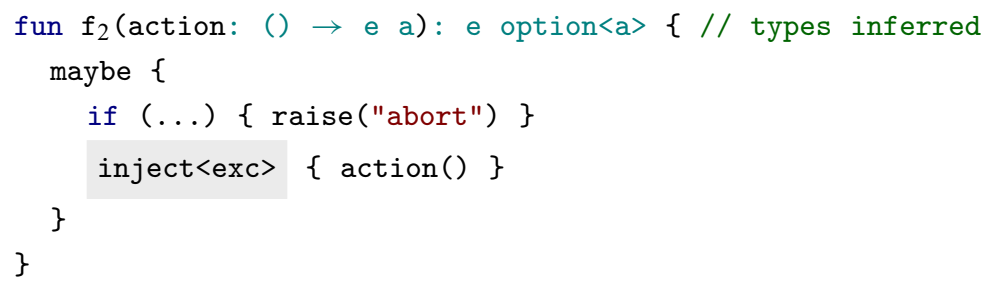

Manually injecting the exc effect into the effect row also has operational content as described by Leijen (2018): the runtime search for the exception handler will skip the maybe handler in $f_{2}$. Now the type of $f_{2}$ truthfully states that it does not handle any effects in e-including any exception effects.

In Effekt, we can directly express the two variants of the function $f$ with different types:

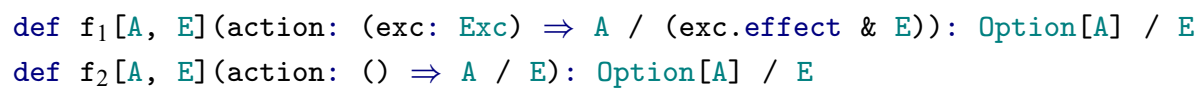

The type of $f_{1}$ makes it clear that action has an unhandled exception effect, handled by $f_{1}$. Furthermore, no inject is necessary to select the right handler, since capabilities are named and passed explicitly.

\subsubsection{Encapsulation in return clauses}

In addition to the implementation of effect operations, in languages like Koka, Eff, or Frank handlers also need to implement a return clause (called unit in the following example):

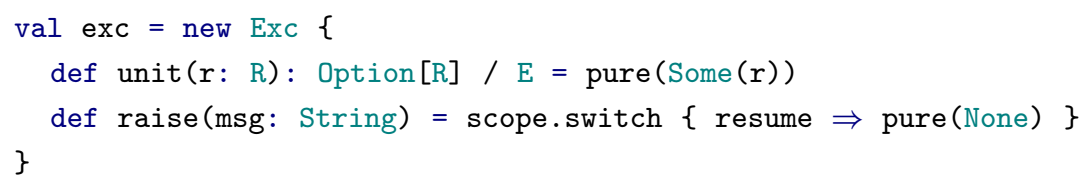

This additional abstraction exists both for historical and technical reasons. Historically, algebraic effect handlers were conceived as a fold over the tree of computation operations (Plotkin \& Pretnar, 2009). Return clauses are required to lift pure values into the domain of computations. In Effekt, it is possible to express return clauses in terms of mapping over the result like: 
handle $\{$ scope $\Rightarrow$ val exc $=\ldots ; \operatorname{prog}(\operatorname{exc})$ flatMap exc.unit $\}$

However, languages like Koka require some form of lifting or inject to make this transformation since effect operations used in the return clause might accidentally be handled by the same handler that has the return clause (Leijen, 2018; Lindley, 2018). Again, this is not an issue in Effekt, since the connection between handler and operations is established explicitly via capability passing. Using capability passing, return clauses are not required to be part of the user interface of handlers while maintaining the same expressive power.

\section{Even more extensible effects}

In previous sections, we have seen how to write simple programs with Effekt (Section 2) and how to establish effect safety (Section 4). In this section, we now give additional examples to evaluate the different dimensions of extensibility gained by embedding Effekt into Scala - a language that supports functional and object-oriented paradigms. In particular, we will see how to compose effect signatures, effect handlers, and effectful programs.

\subsection{Composing effect signatures}

In the introduction, we alluded to the fact that mapping effect signatures and handlers to existing features of object-oriented programming allows us to reuse the abstractions those features offer. In particular, as we will see, mapping signatures to Scala's traits opens up interesting new modularity benefits.

\subsubsection{Extending effect signatures}

Since signatures are traits, we can extend them and add new operations. Here, we extend the Amb trait (defined in Figure 2(a), Section 2) with an additional effect operation choose.

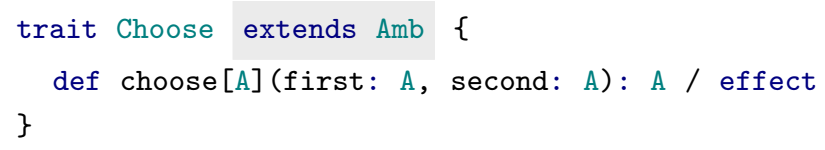

This introduces a subtyping relationship between Amb capabilities and Choose capabilities. A handler for Choose thus can also be used to handle Amb. This cannot be expressed in Koka, for example, where a handler is tied to a single-effect signature and can only handle precisely the effect with this signature.

\subsubsection{Default methods: primitive versus derived effect operations}

Traits in Scala cannot only contain abstract method declarations, but also concrete method implementations. Similarly, our effect signatures cannot only contain abstract operations, but also concrete effect operation implementations, as illustrated in the following example.

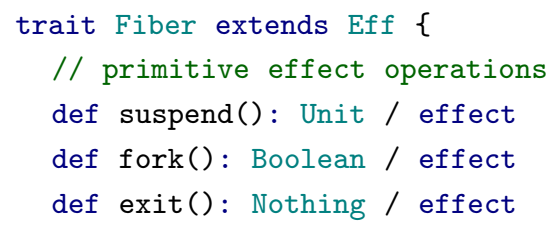




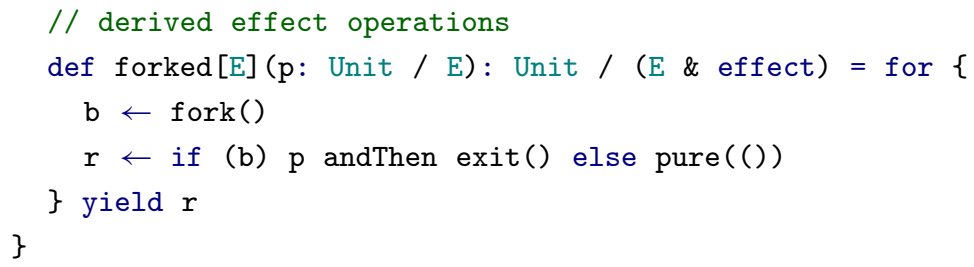

Here, the Fiber effect (Dolan et al., 2015; Leijen, 2017b) for cooperative multitasking has three abstract effect operations that need to be implemented by handlers. The effect operation suspend indicates that the current fiber can be suspended. The effect operation fork is similar to the equally named system call in Unix, spawns a new fiber, and returns true if the code is executed as part of this new fiber. Finally, the effect operation exit terminates the current fiber. In addition to the three abstract effect operations, the effect signature also contains a concrete effect operation forked. The argument computation of type Unit / E represents the code, which is executed as part of the forked fiber. The effect operation forked is implemented in terms of fork and exit. We refer to operations like suspend as primitive effect operation and to operations like forked as derived effect operations. Derived effect operations can be overridden in handler implementations, for example, for efficiency. Furthermore, the derived operation forked does not make any assumptions about the handler implementation. In particular, it does not explicitly capture the continuation with scope but only uses the other effect operations of Fiber. This illustrates an important difference to languages like Koka, where every effect operation always automatically captures the continuation.

\subsubsection{Abstract type members: Effect signatures as module interfaces}

Another particularly interesting example of abstraction reuse is Scala's abstract type members. Mapping effect signatures to Scala traits, they cannot only describe effect operations, but also have (abstract) type members. This opens up interesting ways to structure effect signatures, illustrated by the following signature of the Async effect (Dolan et al., 2017):

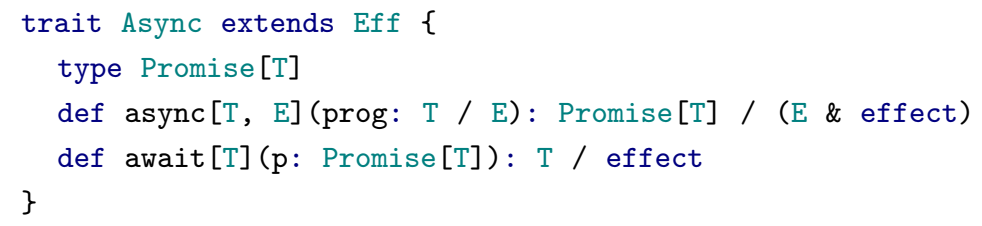

The abstract type member Promise allows handler implementations to choose the representation of promises. The effect signature also declares two effect operations (async and await) that refer to the abstract type. The operation async takes an effectful computation and returns a promise that can be awaited with the effect operation await. In a concrete capability of type Async, the choice of representation for Promise is hidden existentially. Hiding the representation prevents instances of promises to be awaited outside of the corresponding Async-handler.

Example: Asynchronous Programming. Having declared the effect signatures for Async and Fiber we can write effectful programs using these effects: 


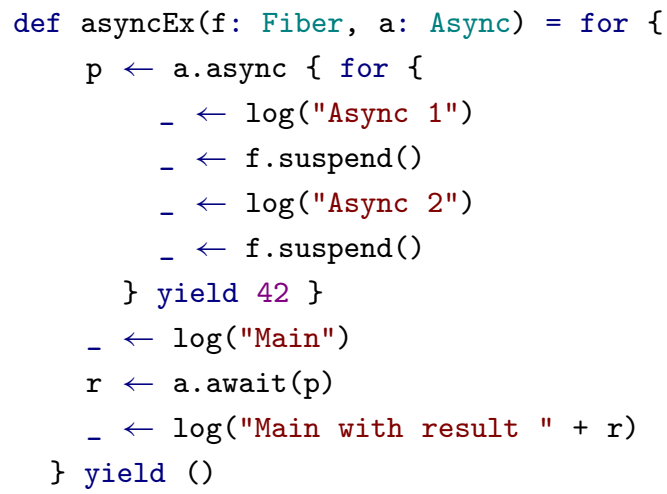

We will see how to run this example after having defined the handlers for Fiber and Async.

\subsubsection{Nested traits: Families of effectful types}

Abstract type members like Promise are not the only way to express a family of effectful types. Traits in Scala can also be nested, and we can refactor the effect signature Async to:

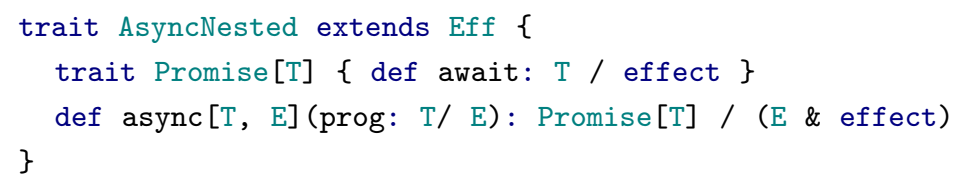

Like in the effect signature of the State effect (Figure 5(b), Section 4), the type effect used by Promise refers to the type member on AsyncNested.

\subsubsection{Mixing effect signatures}

Scala supports mixin composition on traits (Odersky \& Zenger, 2005b) so we can mix independently declared effect signatures. For example, we can mix the two effect signatures for ambiguity and exceptions to obtain a combined effect signature for nondeterminism:

trait Nondet extends Amb with Exc

Furthermore, since traits can contain both abstract and concrete definitions, effect signatures can be mixed to mutually implement primitive (i.e., abstract) effect operations in one signature by derived (i.e., concrete) effect operations in another. This works for both abstract types and abstract methods.

\subsection{Composing effect handlers}

All effect handlers so far were implemented as anonymous inner classes. This style of implementing handlers precludes certain forms of reuse.

\subsubsection{Handlers as traits}

To recover extensibility and reuse, we can express effect handlers as traits. Effects that are required by the implementation can be expressed as abstract value members. For instance, expressing the maybe handler (Figure 2(b)) as a trait looks like: 


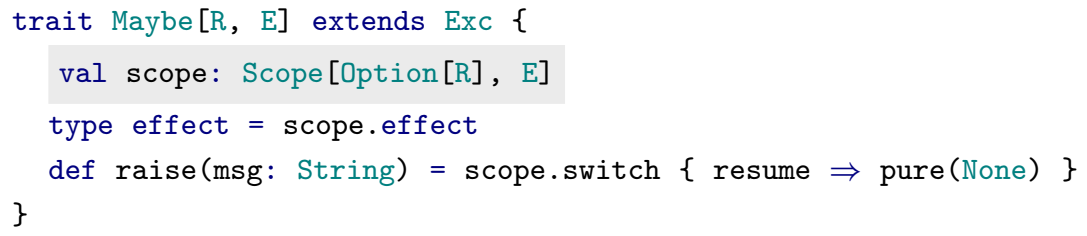

Here we express the dependency on the scope effect as an abstract value member scope. The type of Scope communicates that it needs to be delimited at some optional type. Otherwise, to increase reuse, the Maybe handler is parametric in type $\mathrm{R}$ and the set of effects $E$ at the scope delimiter. Similarly, we can express the handler for ambiguity as a trait:

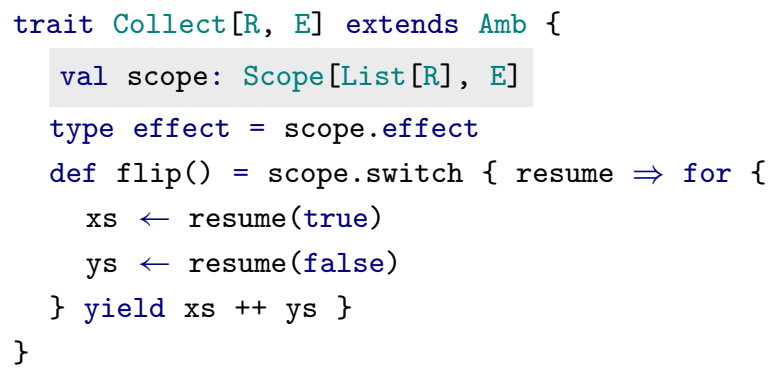

Handling the Amb effect with the Collect handler trait now amounts to constructing a handler instance of Collect and passing it to the program.

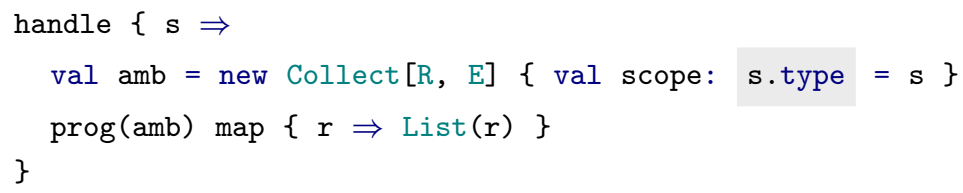

The highlighted type refinement is essential. It allows the type checker to locally unify scope.effect, s.effect, and amb. effect. This way, handle can remove amb. effect from the set of effects of prog. Expressing handlers as traits turns them into reusable, composable, and extensible components. We will now explore the different dimensions of extensibility enabled by this technique.

\subsubsection{The effect expression problem}

Many implementations of libraries and languages for (algebraic) effects and handlers are based on a deep embedding of effect operations. They reify effect operations as alternatives in a sum type and represent effectful computations as a command-response tree. For instance, the flip effect operation would be reified as a constructor of an algebraic data type Amb. Handlers fold over the tree of computation and interpret the reified effect operations by pattern matching on them (Bauer \& Pretnar, 2015; Kiselyov \& Ishii, 2015; Hillerström et al., 2017; Leijen, 2017c; Kiselyov \& Sivaramakrishnan, 2018). To mix programs with different effects means to extend an open union type of reified effect operations.

In contrast, by performing capability passing and representing effect signatures as traits, Effekt builds on a shallow embedding (Hudak, 1998; Carette et al., 2007) of effect operations. Instead of folding over the tree of computation, user programs directly call effect 
operations on the handler. In a language with mixin composition, shallow embeddings can be structured in a pleasingly extensible way (Oliveira \& Cook, 2012). Thus, Effekt has a solution to the expression problem (Wadler, 1998) at its foundation, a property it shares with many other effect handler implementations. For instance, languages like Koka (Leijen, 2014), Frank (Lindley et al., 2017), and Links (Hillerström et al., 2017) are based on row polymorphism (Gaster \& Jones, 1996) and Extensible Effects (Kiselyov et al., 2013; Kiselyov \& Ishii, 2015) are based on open unions (Liang et al., 1995).

We relate extensibility dimensions discussed in the literature on the expression problem to effect handlers (Brachthäuser \& Schuster, 2017) and show how Effekt supports them:

Adding New Handlers. The first dimension of the effect expression problem corresponds to adding a new function definition over the recursive data type in the original expression problem. A central feature of every implementation of effects and handlers is the ability to define a new handler for an existing effect. Effekt supports this feature: users can define a new trait or class that implements an existing effect signature.

Adding New Operations. The second dimension of the effect expression problem corresponds to adding a new variant to the recursive data type in the original expression problem. We can distinguish between adding an operation to an existing effect signature and adding a new effect signature. Effekt supports the modular extension of effect signatures as illustrated by the example trait Choose of Subsection 6.1. Other languages like Koka cannot compose effect signatures. In those languages, it is therefore also not necessary to compose or extend handler implementations. Effekt allows the programmer to extend handler implementations modularly.

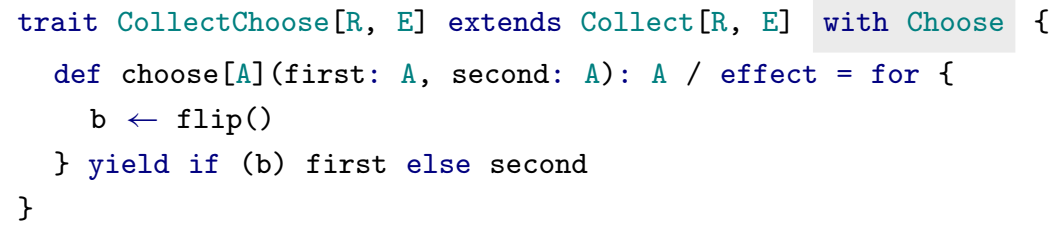

In this example, the handler for the extended effect signature Choose extends the existing Collect handler and only implements the missing effect operation choose. The example also illustrates that we can reuse the implementation of flip to implement choose.

\subsubsection{Mixing handlers-horizontal composition of handlers}

The description of the expression problem has seen many extensions and additional requirements. One additional requirement described by Odersky \& Zenger (2005a) is that the programmer should be able to combine independently developed extensions. For effect handlers, this means to compose two existing effect handlers. This feature might seem unnecessary in the context of effect handlers where handler composition can be expressed by function composition. However, using trait mixin composition to combine two handlers, the handler implementations can share implementation details like private methods and dependencies on other internally used effects. As an example, we define another handler for ambiguity that performs backtracking to compute only the first successful result: 


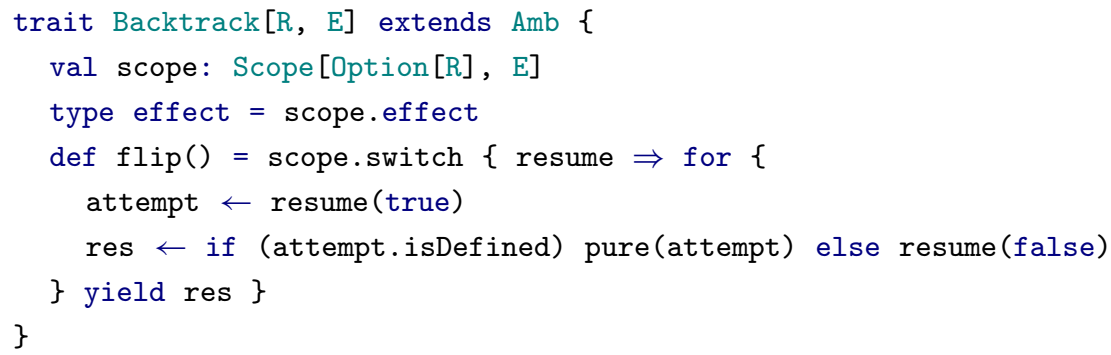

We can implement the Nondet effect simply by mixing the handlers Backtrack and Maybe:

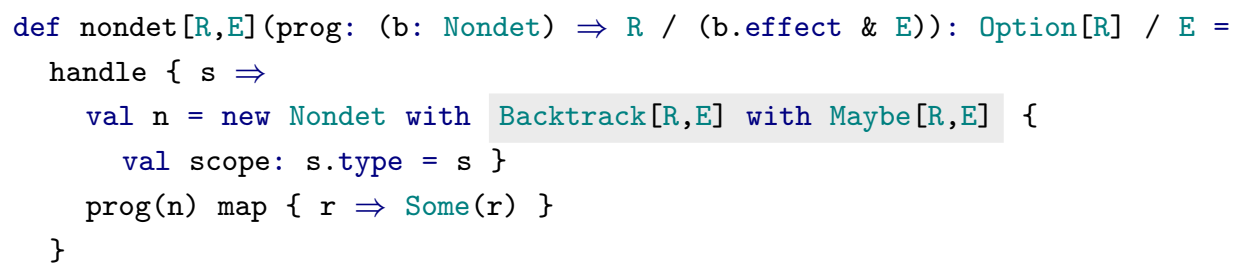

The use of mixin composition is legal, since the two handlers use the same effects. In particular, both the answer-type Option $[R]$ and the set of effects $E$ on scope coincide. Using the handler function nondet, we can handle Exc and Amb simultaneously:

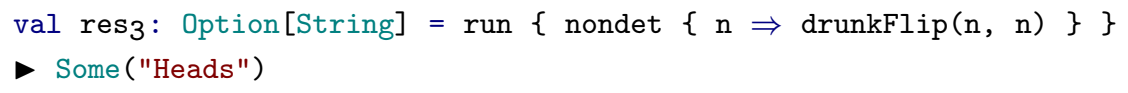

The example illustrates how handlers can be composed horizontally with mixin composition under the condition that they interpret the effects into the same effect domain. Operationally, they share the same scope delimiter. By subtyping, the combined handler can be used to handle both effects. In res 3 , it is passed down twice, once to handle the Amb effect and once to handle the Exc effect.

\subsubsection{Composition over inheritance-vertical composition of handlers}

Effect handlers allow us to locally handle a subset of effects used by a program. To do so, handlers can again use effects in their implementation which are then handled by other handlers. That is, we can compose handlers vertically. However, so far this composition was not particularly interesting. All handlers, that we have so far encountered used the Scope effect and consequently defined type effect $=$ scope. effect.

Different to most other formulations of effect handlers, handlers in Effekt do not have to capture and use the continuation and consequently do not have to use the Scope effect. It is up to the handler implementation to decide. Figure 6 presents an example of such a handler that does not use the scope effect to capture the continuation. Instead, it uses the effects State and Fiber and therefore has the capabilities state and fiber as abstract value members. We use the Fiber effect to fork the computation in async and to implement polling in await. We define Promise to be the type state.Field and implement async to store the result of the asynchronous computation to the field provided by the state capability. The handler function poll takes the two required capabilities to construct an instance of the handler trait Poll: 


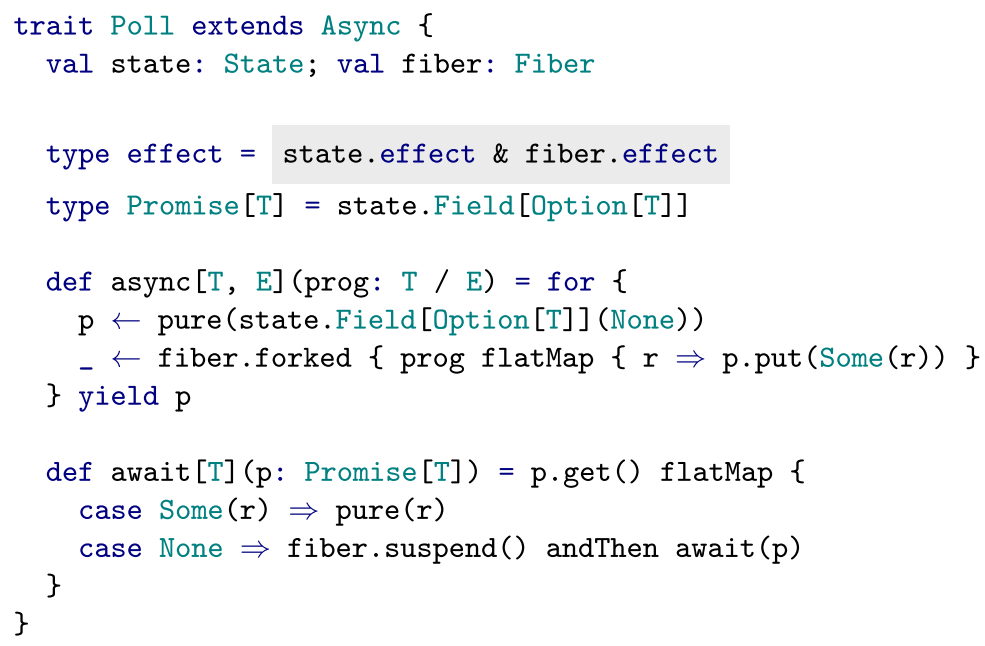

Fig. 6. Handler for the Async effect - using two effects State and Fiber.

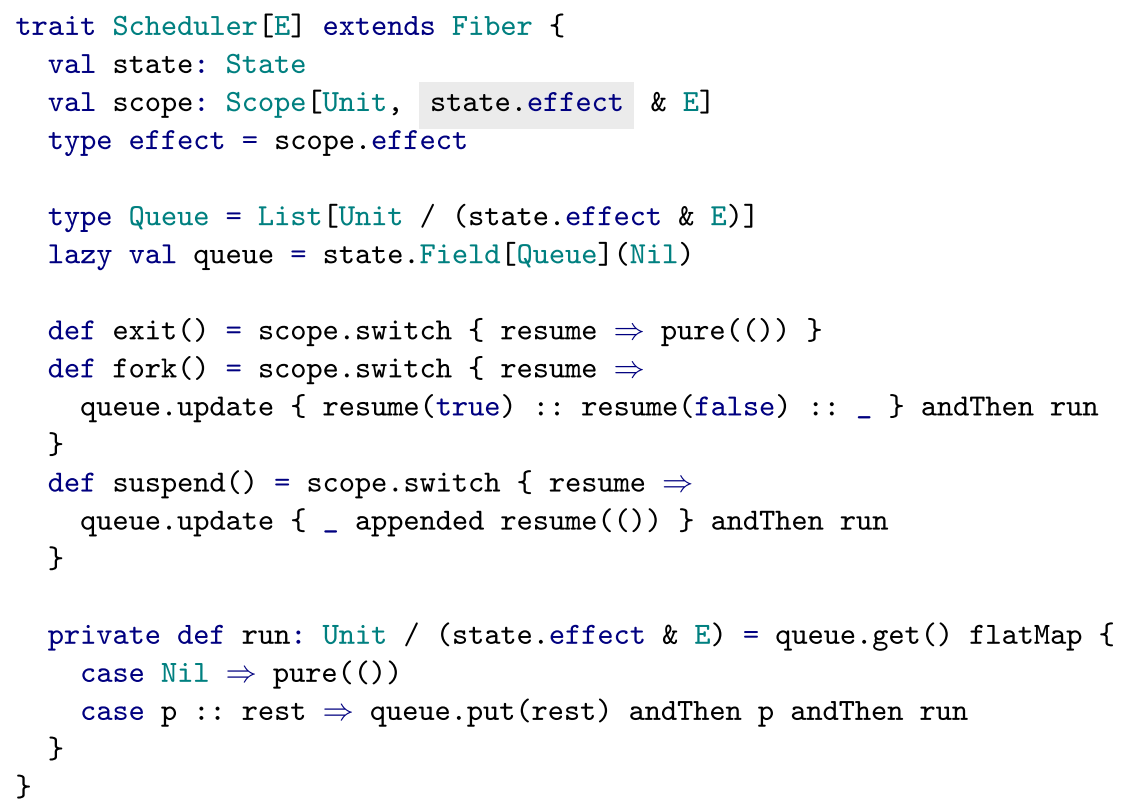

Fig. 7. Handler for the Fiber effect — using the State effect after switching the scope.

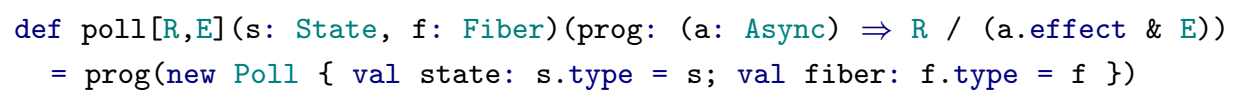

By refining the types of state and fiber to singleton types, the inferred return type of poll is $R$ / ( $E$ \& s.effect \& f.effect). It thus communicates precisely that we implement the Async effect in terms of the given state and fiber capabilities. 


\subsubsection{Vertical composition and scopes}

The Poll handler (Figure 6) uses two effects State and Fiber in its implementation, but does not use the Scope effect to capture the continuation. All the other effect handlers we have seen so far do use the scope effect (e.g., a capability of type Scope [R, E]) but are parametric in the set of outer effects $E$. That is, after switching the scope those handlers did not use any other effects. However, there is another combination of using effects that we have not seen so far: first, switch the scope and then use another effect.

Figure 7 uses this technique to implement the Fiber effect as a round robin scheduler. It requires the two capabilities state and scope. It uses the state capability to maintain a queue of fibers that still needs to be scheduled. Fibers are obtained by capturing the continuation using the scope capability. The difference to the previous examples now is in the type of the scope capability. It informs us that the state effect is available after switching the scope. This is not relevant for the implementation of the effect operation exit which simply discards the continuation. However, the two remaining operations fork and suspend both use the state effect in the body passed to scope.switch.

The type of the scope capability imposes an order on how state and scope need to be handled. The state effect is required to be the outer handler. Not only is this important to be able to use state after switching the scope, it is also important for the semantics of the scheduler: the handler uses state to store a queue of running fibers. The state should be persisted across different fibers, which are forked by resuming once with true and once with false. The handler function for the scheduler directly handles the Scope effect, but leaves the state effect open. The inferred return type of scheduler is thus Unit / (st.effect \& E).

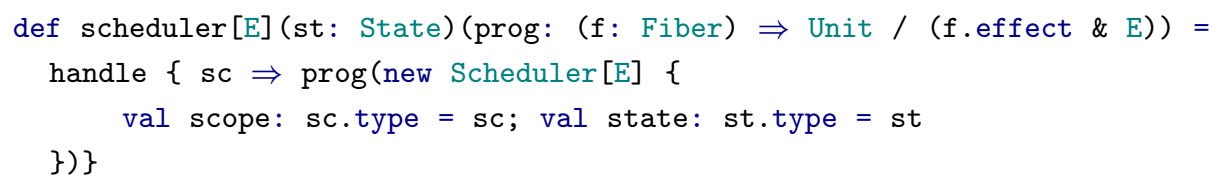

This mode of use is how all effect handlers are implemented in Koka, Eff, and Frank. In those languages, the body of an effect operation is always executed at the call site of the handler, not the operation. Also all effects used in the implementation of the effect operation will be evaluated at the handler call site (i.e., the call to handle in our case). This is essential to encapsulate effects as implementation details and not leak their usage into the call site. As seen with the Poll handler, Effekt offers an alternative to encapsulate effects by employing capability passing and using (path-dependent) abstract type members.

Example: Running Asynchronous Programs. Having defined handlers for the Fiber and Async effects, we can now finally run asyncEx (Subsection 6.1.3) as follows

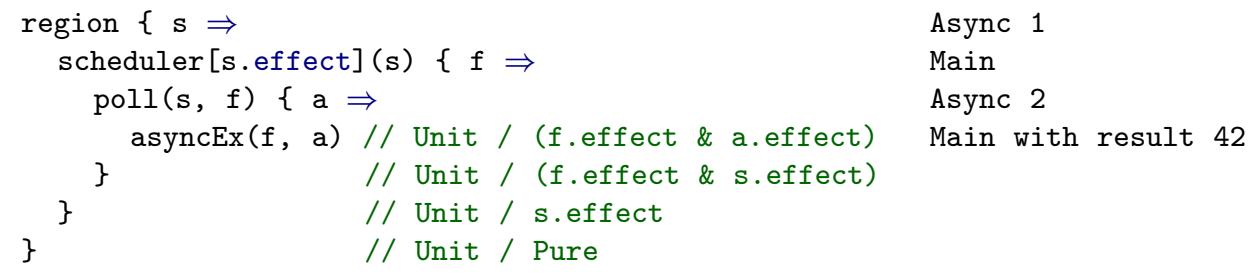

Async 1

Main

Async 2

Main with result 42 
resulting in the output on the right. Type inference is not proficient enough to infer the removal of effects and we need to annotate the effect type s.effect at the call to scheduler.

\subsection{Composing effectful programs}

By passing capabilities explicitly, we are able to select which instance of an effect to use in the presence of multiple instances of the same effect. For example, we can use multiple instances of Fiber in one program to model different thread pools. At the same time, explicit capability passing can be a burden since it introduces manual boilerplate. This is illustrated by the following example that uses the three effects ambiguity, exceptions, and reading from an input stream to model a parser. The parser recognizes an arbitrary number of characters ' $a$ ' followed by a single character ' $b$ '.

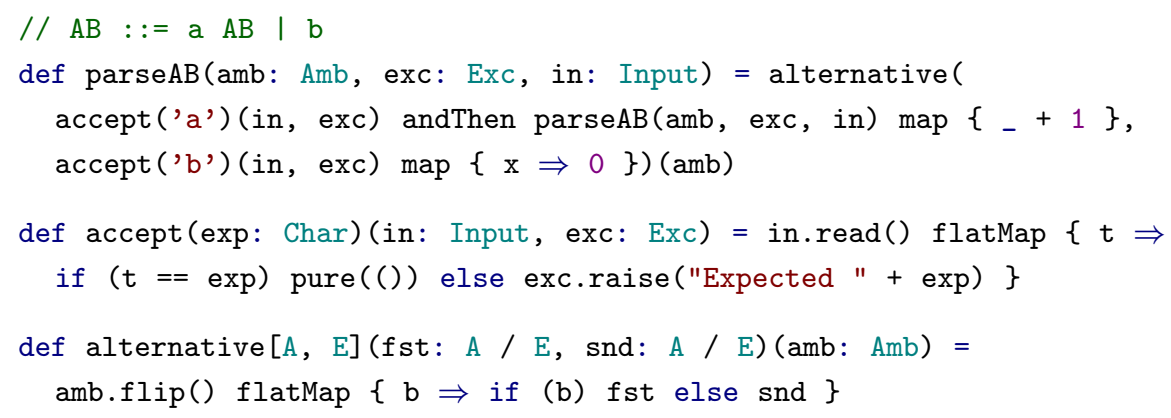

In the example, we use an Input effect that supports reading from an input stream.

trait Input extends Eff $\{\operatorname{def} \operatorname{read}():$ Char / effect $\}$

When composing effectful programs that use different effects, the programmer needs to manually pass the capabilities to the respective function calls. In particular, a function like parseAB that uses other effectful functions (like accept and alternative) needs to take the union of all capabilities required by its dependencies. To call them, the programmer needs to select the correct subset of capabilities and provide them along other arguments. For example, all three capabilities need to be passed to the recursive call.

\subsubsection{Implicits for capability-passing style}

While the overall design of Effekt is largely independent of Scala, there are certain features that ease the use of the library. One such feature is implicit parameters. Implicit parameters (now called "given-clauses" or "contextual parameters" in Scala 3) can help to automatically pass function arguments based on their type (Odersky et al., 2017). This makes implicits a perfect fit for the Effekt library.

To implicitly look up capabilities, for every effect signature, we define functions like:

$\operatorname{def}$ Amb given ( $a$ : Amb): a.type = a

Calling the nullary function Amb implicitly searches for a value of the equally named type in the current scope and returns it. As before, the return type is a singleton type to allow the necessary unification of path-dependent types our effect system relies on. Using these helpers, we can now rewrite the above example to: 


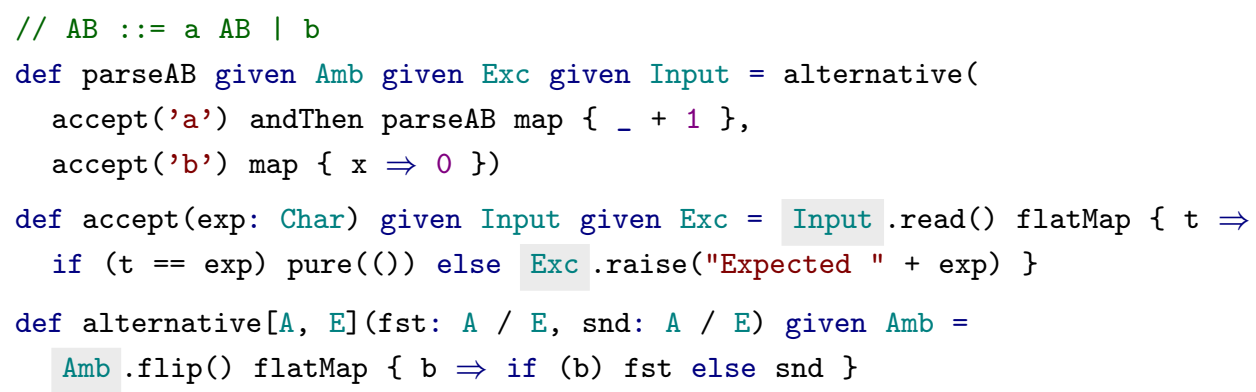

Note how all the arguments to the recursive call to parseAB (and to the functions accept and alternative) are now provided implicitly. The signature of accept informs us that it relies on an instance of Input and an instance of Exc being in scope at the call site. ${ }^{4}$ At the same time, it brings these two instances in scope for the method body, so Input.read and Exc.raise will resolve to method calls on the corresponding implicit argument. Here, Input is the nullary method call to a boilerplate function as defined above. For the purpose of this paper, it is enough to understand that implicit search is performed at compile time, is lexically scoped, and type-directed. Implicit resolution results in a program like the explicit capability-passing variant above. We can also choose to bind implicit parameters to explicit names. In fact, this is necessary for the method parseAB which is recursive and thus requires a type annotation:

def parseAB given (a: Amb) given (e: Exc) given (i: Input)

: Int / (a.effect \& e.effect \& i.effect)

Binding capabilities to names also enables us to fall back to passing them explicitly (e.g., accept ('a') given a given e). This is important to resolve conflicts in case of ambiguous implicits, which would otherwise result in a compile time error.

\subsubsection{Reducing the overhead by composition}

Another strategy to reduce the burden of passing capabilities is by composition. We can define a trait that contains the necessary capabilities as members:

trait Parser \{ val amb: Amb; val exc: Exc; val input: Input \}

Now all three methods can be refactored to only take one (potentially implicit) argument of type Parser, manually projecting the fields where necessary. Interestingly, the type signature of accept then becomes

def accept (exp: Char) (p: Parser): Unit / (p.exc.effect \& p.amb.effect)

This illustrates the flexibility of path-dependent types in Scala. The stable path can have an arbitrary length.

\footnotetext{
4 Since Scala 3, naming implicitly bound variables is optional. The signature thus roughly corresponds to
} def accept (exp: Char) (implicit \$1: Input, \$2: Exc) in Scala 2. 


\subsection{Effect handlers and object orientation}

Effekt is an embedding of effect handlers in a language with support for object-oriented programming. Naturally, the question arises how these two features interact.

Object-oriented programming has a strong focus on encapsulation. In particular, the concrete implementation of an object and its internal state is often hidden behind an interface. That is, the implementation can differ with the granularity of a single object. Another important feature is that objects are first class and typically are stored on the heap. In contrast, effects and handlers are tied to a stack discipline. Effect handler implementations can capture parts of the stack as a continuation, handlers delimit segments of the stack and effect typing asserts that these stack operations are safe, which effects are used by an object's implementation can either be seen as part of the public interface or as a private implementation detail. It is a design decision the programmer should make. However, if the effects used by an object are hidden behind an interface, how can we assert effect safety? For instance, if an object closes over a capability, the object's lifetime needs to be restricted to the capability's lifetime. Otherwise, the use of the capability might not be effect-safe.

The following interface will serve as a running example to discuss possible design choices when safely combining effect handlers with object-oriented programming:

trait Person \{ def greet(other: String): Unit \}

\subsubsection{Alternative 1: Effects as part of the public interface}

An implementation might want to use the following effect to print the greeting.

trait Console extends Eff \{ def print(msg: String): Unit / effect \}

However, the method greet as declared above does not mention the Console effect. Of course, we can change the interface accordingly.

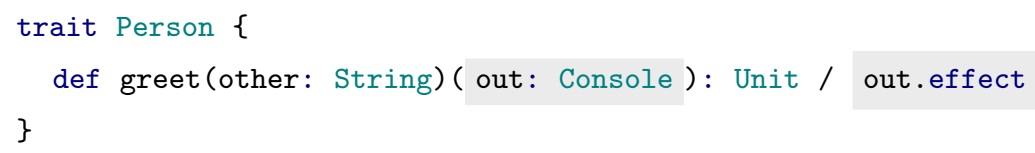

Now, the Console effect is part of the public interface and all implementations of Person can make use of it to implement the method greet. The effect has to be handled by the caller of greet. In this variant, it is possible to have multiple different implementations of Person and store the instances in data structures on the heap.

\subsubsection{Alternative 2: Hiding effects behind an interface}

Changing the interface of Person to mention the effects used by a particular implementation leaks implementation details. This problem also occurs with checked exceptions in Java. We can think of Console as a checked exception that is not mentioned in the interface of greet. Java programmers often resort to wrapping checked exceptions in unchecked ones to work around this problem (Zhang et al., 2016). The exceptions thrown by an implementation can be considered an implementation detail that we might want to encapsulate. In Effekt, we can hide the effects behind an abstract type member effect. 


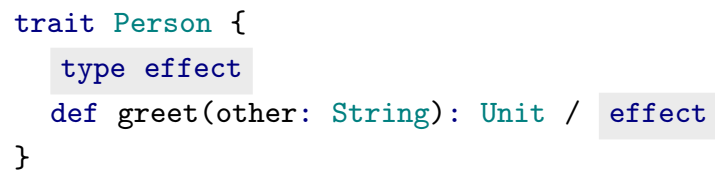

An implementation of Person closes over the effect capabilities, just like a handler does:

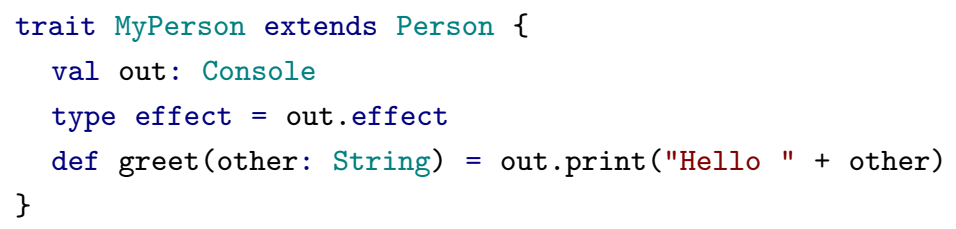

Assuming a handler for the Console effect, in the following example, the lifetime of an instance of MyPerson is now coupled to the lifetime of the capability o.

$$
\text { withConsole }\{0 \Rightarrow \ldots \text { val } p=\text { new MyPerson }\{\text { val out: } 0 . \text { type }=0\} \ldots\}
$$

For instance, the object $\mathrm{p}$ must not be used outside the scope of withConsole, which is ensured by our effect system: out.effect is an abstract type that only unifies with this one particular o created at the call to withConsole.

To be able to eventually handle the effects used by the implementation, users always need to have stable paths to an object.

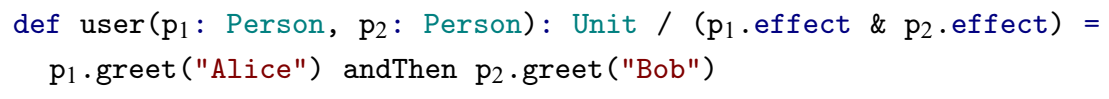

In this example $p_{1}$ and $p_{2}$ are arguments of method user and have stable paths that can be referred to in the return type. In general, path stability excludes objects from being stored in mutable references or containers like lists. While we can store $\mathrm{p}_{1}$ in a mutable variable, the effect system will prevent us from calling any effectful methods on it.

\subsubsection{Alternative 3: Grouping objects by their effect implementations}

Alternative 1 requires all objects to use the same effects in their implementation and Alternative 2 allows each object to individually differ in their effect implementation. Both solutions also have drawbacks: the former constrains the implementer while the latter imposes restrictions on the user. As a compromise between the two, we can generalize over the effect implementation and thereby group objects by their effect implementations.

trait Person[E] \{ def greet(other: String): Unit / E \}

Like with abstract type members, implementing classes can instantiate $E$ to the desired implementation effects. Like with the first alternative, objects of type Person [Console] leak the implementation detail that they use the Console effect in their implementation.

Programs using instances of Person can be polymorphic in the effect type:

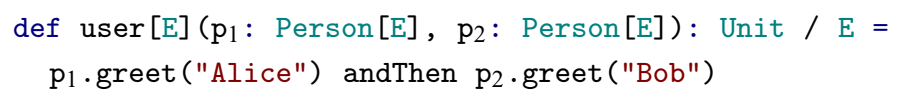

While we now can store objects of type Person [E] in lists of type List [Person[E]], this requires all instances to have the same effect implementation. Furthermore, instances of 
Person [o.effect] are still coupled to the lifetime of the capability o. An implementation that depends on more than one effect can only be used in the intersection of the respective handler regions.

\section{Related work}

In this section, we discuss closely related work. In particular, we compare our approach of capability passing to other implementations of effect handlers and relate our effect handlers design for object-oriented languages to others.

Shallow Embedding of Effect Operations. Many implementations of libraries and languages for effect handlers are based on a deep embedding of effect operations. In contrast, as pointed out in Section 6.2.2, by performing capability passing, Effekt builds on a shallow embedding of effect operations. Similarly, Kammar et al. (2013) base their library implementation of algebraic effect handlers on Haskell's type classes, effectively performing a shallow embedding. Using type classes and the associated dictionary passing helps Kammar et al. (2013) to achieve good performance results, since it prevents the materialization of constructors for effect operations. It also avoids any search for the matching handler implementation in some kind of handler stack, as it is done in Koka (Leijen, 2017a, c).

Continuations and Delimiters. Our implementation of delimited control is based on Dybvig et al. (2007), who in turn present a monadic implementation of a variant of multi-prompt delimited control by Gunter et al. (1995). Dybvig et al. (2007) present a very general framework that can express many different control operators. In particular, capturing the continuation with their control operator withSubCont removes both the corresponding outer delimiter and excludes it from the captured continuation. In contrast, our effect operation switch captures and removes the continuation up to and including the corresponding delimiter handle. It is thus operationally closer to shift ${ }_{0}$ by Danvy \& Filinski (1989, Appendix C) and spawn by Hieb \& Dybvig (1990) as illustrated by the following equation:

def $\operatorname{spawn}($ body) $=$ handle $\{\mathrm{s} \Rightarrow \operatorname{body}(\mathrm{s} . \mathrm{switch})\}$

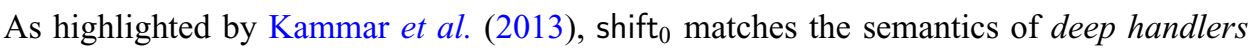
where the same effect is already handled in the continuation. Materzok \& Biernacki (2011) present an effect system for shift $t_{0}$ that supports answer-type modification, while our effectsafe version of Control does not. In turn, Effekt allows handlers to switch to a specific scope, while shift $t_{0}$ always captures the continuation up to the closest delimiter.

Effect-Safe Multi-Prompt Delimited Control. Like our presentation in Section 3, Dybvig et al. (2007) guarantee answer-type safety by indexing prompts with the expected answer type. Furthermore, they use rank-2 types to prevent prompts from being used across different instances of run. But, as they observe, this is not enough to achieve effect safety, which they explicitly leave to future work. We generalize the idea of region safety and guarantee that capabilities cannot be used outside of the scope that they are 
created in. Instead of rank-2 types, we use abstract type members. This lets us easily nest scopes using intersection types. In general, Scala has better support for path-dependent function types than for rank-2 types. For instance, we can use the lambda syntax (e.g., $\mathrm{amb} \Rightarrow \mathrm{amb} . \mathrm{flip}())$, which is not possible with rank-2 types that need to be instantiated as anonymous inner classes. Compared to path-dependent function types, the use of rank-2 types is thus more verbose and hinders type inference.

Region Safety for Effects. Many languages with effect handlers base their effect system on some form of row polymorphism. Prominent examples are Koka (Leijen, 2014), Frank (Lindley et al., 2017), and Links (Hillerström \& Lindley, 2016). In contrast, effect-safe library embeddings like Extensible Effects (Kiselyov et al., 2013; Kiselyov $\&$ Ishii, 2015) use various forms of open union types to track the list of unhandled effects. In Effekt, we index effectful computations with an intersection of all capabilities used by a computation. This way, capabilities cannot be used outside of their handler region.

Region Safety for Resources. To achieve resource safety, Kiselyov \& Shan (2008) generalize from a single region (Moggi \& Sabry, 2001) to multiple nested regions. They achieve region polymorphism and region subtyping together with good type inference for their library in Haskell. On the type level, they represent nested regions as nesting of a monad transformer while we represent nested delimiters by an intersection of abstract type members. To achieve region polymorphism, they reuse Haskell's polymorphism and to achieve region subtyping they use Haskell's type class instance search. In contrast, to achieve effect polymorphism, we reuse Scala's polymorphism and to achieve subtyping, we reuse subtyping for intersection types built into Scala.

Region Safety for Variable Scopes. Parreaux et al. (2017) apply a strategy very similar to ours to guarantee scope safety in type-safe meta-programming. The type parameter Ctx of their type Code [+Typ, -Ctx] is used to track the set of free variables:

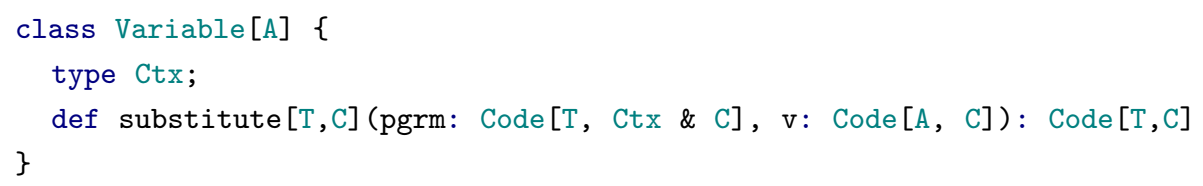

As can be seen from the type of substitute, substitution of free variables removes Ctx from the intersection type and thus corresponds to handling of effects in Effekt.

Capability Passing. Like in this paper, Osvald et al. (2016) perform capability passing in Scala: a capability serves as a constructive proof that the holder is entitled to use the actions associated with the capability. To prevent leaking of capabilities, Osvald et al. (2016) introduce a type-based escape analysis as an alternative approach to traditional effect systems: arguments to functions can be marked as second class (or "local"). The type checker then guarantees that the capability cannot leave the dynamic scope of the function call. Liu (2016) presents a different approach to capability-based effect safety, by distinguishing between functions that can capture capabilities and others that cannot (called "stoic"). In our design of Effekt, we adopt the capability passing of Osvald et al. (2016). Second-class 
values might be an interesting alternative to the effect system presented in this paper. However, they are not available in Scala, while our library can readily be used.

Abstraction-Safe Effect Handlers via Tunneling. The dynamic and static semantics of Effekt is closely related to $\lambda_{\text {ऽ }}$ presented by Zhang \& Myers (2019). As in previous versions of Effekt (Brachthäuser \& Schuster, 2017), capabilities in $\lambda_{\Omega \triangleleft}$ are pairs of a label and the handler implementation. Also like in Effekt, they are explicitly passed to the use site of the effect operation. Handling an effect introduces a fresh label. Like scope capabilities in Effekt, the label is used on the term level to delimit the scope of captured continuations. The label is used on the type level to track the set of unhandled effects, similar to the type member effect in the present paper. Due to the embedding of Effekt in Scala, scope capabilities are first class while labels in $\lambda_{\text {su }}$ are not first class. Instead, the binding of a label by means of try and the use of a label in a handler implementation is statically scoped. For better comparison of $\lambda_{\sqrt{ } \mathrm{s}}$ and Effekt, Appendix A provides a more direct embedding into Scala.

Effect Handlers and Object-Oriented Programming. In earlier work on Effekt (2017; 2018), we started to explore the combination of effect handlers and object orientation. However, those versions of Effekt did not guarantee effect safety. The present paper shows how to add effect safety to Effekt and support effect polymorphism. Indexing the monad for delimited control with the set of used effects is essential to guarantee effect safety. It is not immediate to us how the effect system can be embedded in a direct style version of the library (Brachthäuser et al., 2018) because there is no monadic type that could carry the set of used effects. As highlighted in Section 6, effect-safe programming with effect handlers in a language with objects comes with new challenges-mediating encapsulation and flexible use of objects. Inostroza \& van der Storm (2018) also combine effect handlers and object orientation in the language JEff. In JEff, the continuation takes an updated copy of the effect handler as additional argument. Parametrizing continuations by the handler instances can model dynamically scoped state (Kiselyov et al., 2006) and also allows handlers to change their implementation for the rest of the computation, similar to shallow handlers. The effect system of JEff does not feature effect polymorphism and hence problems with effect encapsulation and effect parametricity do not arise.

\section{Conclusion}

In this article, we presented Effekt, a monadic library for programming with effect handlers in Scala that features effect polymorphism, effect subtyping, and effect safety. We use intersection types and path-dependent types to track the set of effects a program might use. This allows us to directly reuse Scala's support for polymorphism for effect polymorphism and Scala's support for subtyping for effect subtyping. Combining effect handlers with object-oriented programming both offers new ways to modularize effectful programs but also comes with new challenges.

\section{Conflicts of Interest}

None 


\section{References}

Amin, N. \& Tate, R. (2016) Java and Scala's type systems are unsound: The existential crisis of null pointers. In Proceedings of the Conference on Object-Oriented Programming, Systems, Languages and Applications. New York, NY, USA: ACM, pp. 838-848.

Bauer, A. \& Pretnar, M. (2015) Programming with algebraic effects and handlers. J. Log. Alg. Methods Program. 84(1), 108-123.

Biernacki, D., Piróg, M., Polesiuk, P., \& Sieczkowski, F. (2017) Handle with care: Relational interpretation of algebraic effects and handlers. Proc. ACM Program. Lang. 2(POPL), 8:1-8:30.

Biernacki, D., Piróg, M., Polesiuk, P. \& Sieczkowski, F. (2019) Abstracting algebraic effects. Proc. ACM Program. Lang. 3(POPL), 6:1-6:28.

Brachthäuser, J. I. \& Schuster, P. (2017) Effekt: Extensible algebraic effects in Scala (short paper). In Proceedings of the International Symposium on Scala. New York, NY, USA: ACM.

Brachthäuser, J. I., Schuster, P. \& Ostermann, K. (2018) Effect handlers for the masses. Proc. ACM Program. Lang. 2(OOPSLA), 111:1-111:27.

Carette, J., Kiselyov, O. \& Shan, C.-C. (2007) Finally tagless, partially evaluated. In Proceedings of the Asian Symposium on Programming Languages and Systems. LNCS, vol. 4807. Berlin, Heidelberg: Springer, pp. 222-238.

Convent, L., Lindley, S., McBride, C. \& McLaughlin, C. (2019) Doo Bee Doo Bee Doo. Technical report. The University of Edinburgh.

Danvy, O. \& Filinski, A. (1992) Representing control: A study of the CPS transformation. Math. Struct. Comput. Sci. 2(4), 361-391.

Danvy, O. \& Filinski, A. (1989) A functional abstraction of typed contexts. Diku rapport 89/12, diku, University of Copenhagen.

Danvy, O. \& Filinski, A. (1990) Abstracting control. In Proceedings of the Conference on LISP and Functional Programming. New York, NY, USA: ACM, pp. 151-160.

Dolan, S., Eliopoulos, S., Hillerström, D., Madhavapeddy, A., Sivaramakrishnan, K. C. \& White, L. (2017) Concurrent system programming with effect handlers. In Proceedings of the Symposium on Trends in Functional Programming. LNCS, vol. 10788. Springer.

Dolan, S., White, L. \& Madhavapeddy, A. (2014) Multicore OCaml. In OCaml Workshop.

Dolan, S., White, L., Sivaramakrishnan, K. C., Yallop, J. \& Madhavapeddy, A. (2015) Effective concurrency through algebraic effects. In OCaml Workshop.

Dybvig, R. K., Peyton J., Simon L. \& Sabry, A. (2007) A monadic framework for delimited continuations. J. Funct. Program. 17(6), 687-730.

Felleisen, M. (1988) The theory and practice of first-class prompts. In Proceedings of the Symposium on Principles of Programming Languages. New York, NY, USA: ACM, pp. 180-190.

Forster, Y., Kammar, O., Lindley, S. \& Pretnar, M. (2017) On the expressive power of userdefined effects: Effect handlers, monadic reflection, delimited control. Proc. ACM Program. Lang. 1(ICFP), 13:1-13:29.

Friedman, D. P., Haynes, C. T. \& Kohlbecker, E. (1984) Programming with continuations. In Program Transformation and Programming Environments, Pepper, P. (ed), Berlin, Heidelberg: Springer-Verlag.

Gaster, B. R. \& Jones, M. P. (1996) A Polymorphic Type System for Extensible Records and Variants. Technical report NOTTCS-TR-96-3.

Gunter, C. A., Rémy, D. \& Riecke, J. G. (1995) A generalization of exceptions and control in ML-like languages. In Proceedings of the Conference on Functional Programming Languages and Computer Architecture. New York, NY, USA: ACM, pp. 12-23.

Haller, P. \& Loiko, A. (2016) LaCasa: Lightweight affinity and object capabilities in Scala. In Proceedings of the Conference on Object-Oriented Programming, Systems, Languages and Applications. New York, NY, USA: ACM, pp. 272-291.

Hieb, R. \& Dybvig, R. K. (1990) Continuations and concurrency. In Proceedings of the Second ACM SIGPLAN Symposium on Principles \& Practice of Parallel Programming. PPOPP'90. New York, NY, USA: ACM, pp. 128-136. 
Hieb, R., Dybvig, R. K. \& Anderson III, C. W. (1994) Subcontinuations. Lisp Symb. Comput. 7(1), 83-110.

Hillerström, D. \& Lindley, S. (2016) Liberating effects with rows and handlers. In Proceedings of the Workshop on Type-Driven Development. New York, NY, USA: ACM.

Hillerström, D., Lindley, S., Atkey, B. \& Sivaramakrishnan, K. C. (2017) Continuation passing style for effect handlers. In Formal Structures for Computation and Deduction, LIPIcs, vol. 84. Schloss Dagstuhl-Leibniz-Zentrum für Informatik.

Hudak, P. (1998) Modular domain specific languages and tools. In Proceedings of the Conference on Software Reuse. IEEE Computer Society, pp. 134-142.

Inostroza, P. \& van der Storm, T. (2018). JEff: Objects for effect. In Proceedings of the 2018 ACM SIGPLAN International Symposium on New Ideas, New Paradigms, and Reflections on Programming and Software. Onward! 2018. New York, NY, USA: ACM.

Johnson, G. F. \& Duggan, D. (1988). Stores and partial continuations as first-class objects in a language and its environment. In Proceedings of the Symposium on Principles of Programming Languages. New York, NY, USA: ACM, pp. 158-168.

Kammar, O., Lindley, S. \& Oury, N. (2013) Handlers in action. In Proceedings of the International Conference on Functional Programming. New York, NY, USA: ACM, pp. 145-158.

Kiselyov, O. \& Ishii, H. (2015) Freer monads, more extensible effects. In Proceedings of the Haskell Symposium. New York, NY, USA: ACM, pp. 94-105.

Kiselyov, O. \& Shan, C.-c. (2008) Lightweight monadic regions. In Proceedings of the Haskell Symposium. Haskell'08. New York, NY, USA: ACM.

Kiselyov, O. \& Sivaramakrishnan, K. C. (2016). Eff directly in OCaml. In ML Workshop.

Kiselyov, O. \& Sivaramakrishnan, K. C. (2018) Eff directly in OCaml. In Proceedings of the ML Family Workshop/OCaml Users and Developers Workshops, Asai, K. \& Shinwell, M. (eds). Electronic Proceedings in Theoretical Computer Science, vol. 285. Open Publishing Association, pp. 23-58.

Kiselyov, O., Sabry, A. \& Swords, C. (2013) Extensible effects: An alternative to monad transformers. In Proceedings of the Haskell Symposium. New York, NY, USA: ACM, pp. 59-70.

Kiselyov, O., Shan, C.-c. \& Sabry, A. (2006) Delimited dynamic binding. In Proceedings of the International Conference on Functional Programming. New York, NY, USA: ACM, pp. 26-37.

Kobori, I., Kameyama, Y. \& Kiselyov, O. (2016) Answer-type modification without tears: Promptpassing style translation for typed delimited-control operators. arxiv preprint arxiv:1606.06379.

Launchbury, J. \& Sabry, A. (1997) Monadic state: Axiomatization and type safety. In Proceedings of the International Conference on Functional Programming. ICFP'97. New York, NY, USA: ACM, pp. 227-238.

Leijen, D. (2014) Koka: Programming with row polymorphic effect types. In Proceedings of the Workshop on Mathematically Structured Functional Programming.

Leijen, D. (2016) Algebraic Effects for Functional Programming. Technical reportt MSR-TR-201629. Microsoft Research technical report.

Leijen, D. (2017a) Implementing algebraic effects in C. In Proceedings of the Asian Symposium on Programming Languages and Systems. Cham, Switzerland: Springer International Publishing, pp. 339-363.

Leijen, D. (2017b). Structured asynchrony with algebraic effects. In Proceedings of the Workshop on Type-Driven Development. New York, NY, USA: ACM, pp. 16-29.

Leijen, D. (2017c). Type directed compilation of row-typed algebraic effects. In Proceedings of the Symposium on Principles of Programming Languages. New York, NY, USA: ACM, pp. 486-499.

Leijen, D. (2018). First class dynamic effect handlers: Or, polymorphic heaps with dynamic effect handlers. In Proceedings of the Workshop on Type-Driven Development. New York, NY, USA: ACM, pp. 51-64. 
Liang, S., Hudak, P. \& Jones, M. (1995)Monad transformers and modular interpreters. In Proceedings of the Symposium on Principles of Programming Languages. New York, NY, USA: ACM, pp. 333-343.

Lindley, S. (2018) Encapsulating effects. Dagstuhl Reports, 8(4).

Lindley, S., McBride, C. \& McLaughlin, C. (2017) Do be do be do. In Proceedings of the Symposium on Principles of Programming Languages. New York, NY, USA: ACM, pp. 500-514.

Liu, F. (2016) A Study of Capability-Based Effect Systems. M.Phil. thesis, École Polytechnique Fédérale de Lausanne, Switzerland.

Materzok, M. \& Biernacki, D. (2011) Subtyping delimited continuations. In Proceedings of the International Conference on Functional Programming. New York, NY, USA: ACM, pp. 81-93.

Moggi, E. \& Sabry, A. (2001) Monadic encapsulation of effects: A revised approach (extended version). J. Funct. Program. 11(6), 591-627.

Odersky, M., Blanvillain, O., Liu, F., Biboudis, A., Miller, H. \& Stucki, S. (2017) Simplicitly: Foundations and applications of implicit function types. Proc. ACM Program. Lang. 2(POPL), 42:1-42:29.

Odersky, M. \& Zenger, M. (2005a) Independently extensible solutions to the expression problem. In Proceedings of the Workshop on Foundations of Object-Oriented Languages.

Odersky, M. \& Zenger, M. (2005b) Scalable component abstractions. In Proceedings of the Conference on Object-Oriented Programming, Systems, Languages and Applications. New York, NY, USA: ACM, pp. 41-57.

Oliveira, B. C. d. S., \& Cook, W. R. (2012) Extensibility for the masses: Practical extensibility with object algebras. In Proceedings of the European Conference on Object-Oriented Programming. LNCS, vol. 7313. Springer, pp. 2-27.

Osvald, L., Essertel, G., Wu, X., Alayón, L. I. G.\& Rompf, T. (2016) Gentrification gone too far? affordable 2nd-class values for fun and (co-) effect. In Proceedings of the Conference on ObjectOriented Programming, Systems, Languages and Applications. New York, NY, USA: ACM, pp. 234-251.

Parreaux, L., Voizard, A., Shaikhha, A. \& Koch, C. E. (2017). Unifying analytic and statically-typed quasiquotes. Proc. ACM Program. Lang. 2(POPL), 13:1-13:33.

Piróg, M., Polesiuk, P. \& Sieczkowski, F. (2019) Typed equivalence of effect handlers and delimited control. In Formal Structures for Computation and Deduction. LIPIcs. Schloss Dagstuhl-LeibnizZentrum für Informatik, pp. 30:1-30:16.

Plotkin, G. \& Power, J. (2003) Algebraic operations and generic effects. Appl. Categor. Struct. 11(1), 69-94.

Plotkin, G. \& Pretnar, M. (2009) Handlers of algebraic effects. In European Symposium on Programming. Springer-Verlag, pp. 80-94.

Plotkin, G. D. \& Pretnar, M. (2013) Handling algebraic effects. Log. Methods Comput. Sci. 9(4), $1-36$.

Rapoport, M. \& Lhoták, O. (2019). A path to DOT: formalizing fully-path-dependent types. Corr, abs/1904.07298.

Sitaram, D. \& Felleisen, M. (1990) Control delimiters and their hierarchies. Lisp Symb. Comput. 3(1), 67-99.

Wadler, P. (1998). The expression problem. Note to Java Genericity mailing list.

Wright, A. K. \& Felleisen, M. (1994) A syntactic approach to type soundness. Inf. Comput. 115(1), 38-94.

Wu, N., Schrijvers, T. \& Hinze, R. (2014) Effect handlers in scope. In Proceedings of the Haskell Symposium. Haskell'14. New York, NY, USA: ACM, pp. 1-12.

Zhang, Y. \& Myers, A. C. (2019) Abstraction-safe effect handlers via tunneling. Proc. ACM Program. Lang. 3(POPL), 5:1-5:29.

Zhang, Y., Salvaneschi, G., Beightol, Q., Liskov, B. \& Myers, A. C. (2016) Accepting blame for safe tunneled exceptions. Proceedings of the Conference on Programming Language Design and Implementation. New York, NY, USA: ACM, pp. 281-295. 


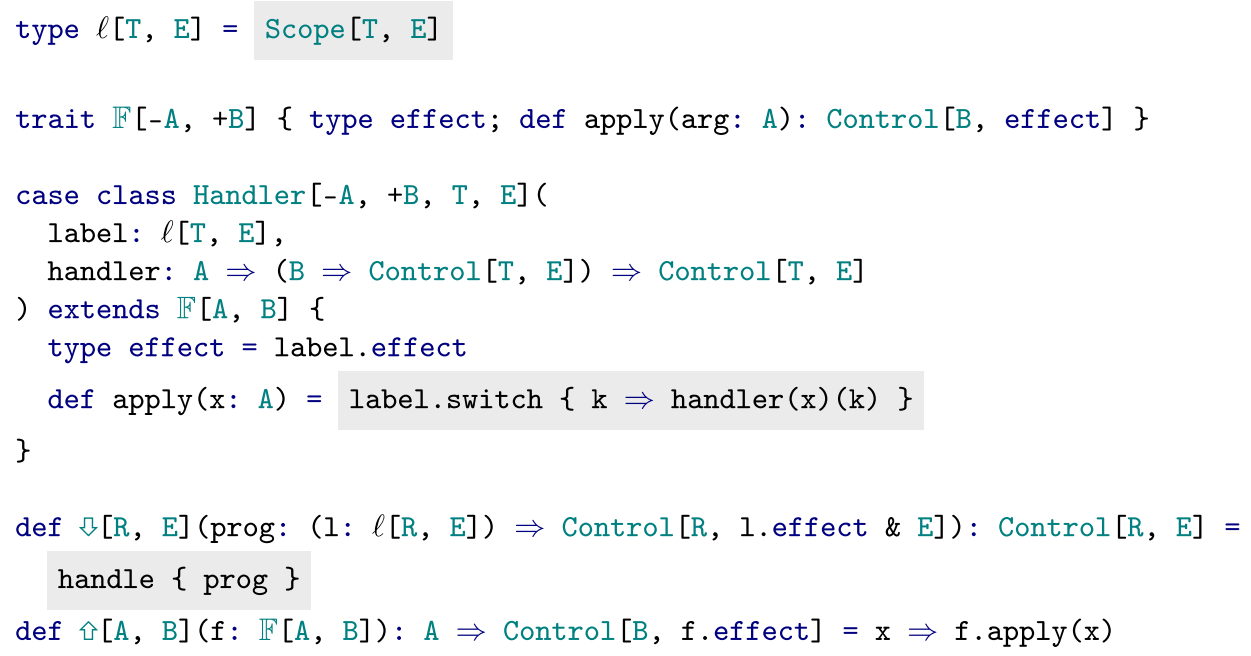

Fig. 8. Using Control to embed the $\lambda_{\Omega}$ calculus into Scala.

\section{Appendix}

\section{Embedding the Tunneling Calculus}

The effect system as presented in this paper is heavily influenced by the one of $\lambda_{\Omega \diamond}$ (Zhang $\&$ Myers, 2019). To ensure effect safety, Zhang \& Myers use a simple form of dependent types: Using an effect handler $\mathrm{h}$ introduces $\mathrm{h} . \mathrm{lbl}$ in the effect type which is effectively a set of labels. The dependent label corresponds to the abstract type member $\mathrm{h}$. effect in Effekt. Like in Effekt, this dependent effect type can only be discharged by the very same delimiter (denoted by $\nabla^{\ell} t$ ) that introduced the label. Zhang \& Myers formalize $\lambda_{\Omega}$ and formally show effect parametricity. However, they do not provide an implementation of their calculus. We use intersection types and path dependent types to encode the ideas of the $\lambda_{\Omega \text { }}$ effect system and thereby make Effekt effect safe.

To facilitate comparison of $\lambda_{\text {s仓 }}$ with Effekt, Figure 8 embeds the $\lambda_{\text {s仓 }}$ calculus (Zhang $\&$ Myers, 2019) in Scala. We immediately use the Control monad and the Scope effect to express the operational semantics. We just present the practical embedding into Scala and leave a formal translation and corresponding soundness proofs to future work. However, assuming a sound subset of Scala that corresponds to $\lambda_{5}$, we conjecture that our embedding faithfully models the calculus by Zhang \& Myers. In particular, the restriction to a subset of Scala excludes the use of mutable state, recursive function definitions, recursive data-types, and exceptions. Also effect signatures have to be declared on the top level and should not use mixin composition, type members, subtyping, or any other advanced Scala feature.

\section{Example}

To ease comparison with the original calculus, we use $\ell$ as the type of labels and $\mathbb{F}$ as the type of effect signatures. Effect signatures $\mathbb{F}[A, B]$ only declare a single-effect operation with argument type A and return type B. For example, we can express the signatures of the ambiguity and exception effect as: 


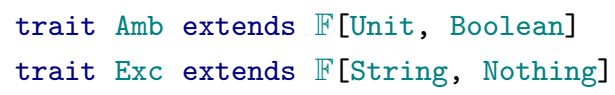

Handlers, modeled by the case class Handler, are pairs of labels and effect implementations. In this style, the handler for expressions can be expressed as:

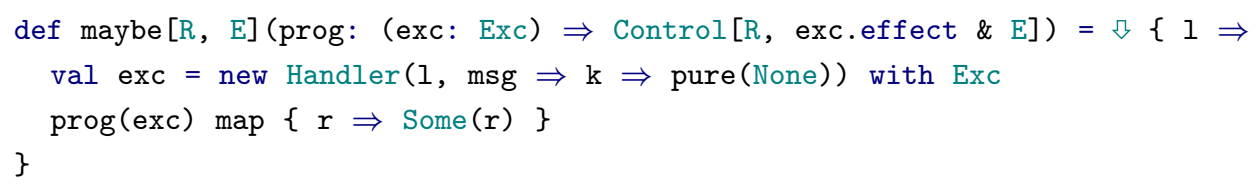

Calling an effect operation amounts to calling $仓$ on the handler:

$$
\text { maybe }\{\mathrm{h} \Rightarrow \ldots \text { i }(\mathrm{h}) \text { ("Failed!") } \ldots\}
$$

While in $\lambda_{\Omega}$, $仑$ performs the continuation capture, in the embedding we perform the capturing in the implementation of Handler.apply by means of label.switch. This is necessary to have the available answer types ( $\mathrm{T}$ and $\mathrm{E}$ in scope). We use subtyping Handler $<: \mathbb{F}$ to existentially hide the answer types and other implementation details of Handler when passing capabilities of type $\mathbb{F}$.

Refinement to Singleton Types. In the presentation of Effekt in this paper, we always performed explicit refinement to singleton types to establish type equalities of type members. Similarly, to prevent widening of the label singleton type to $\ell$, the actual signature of Handler is:

case class Handler $[-A,+B, T, E, L<: \ell[T, E]]($ label: L, ...)

Additionally, at construction site, the type parameter L has to be explicitly provided as singleton type 1. type:

val $\mathrm{h}=$ Handler $[\mathrm{A}, \mathrm{B}, \mathrm{T}, \mathrm{E}, \mathrm{l}$.type] (l, ???). effect

This establishes the type equality between 1 . effect and h.effect. 